\title{
Diagnostics of energetic electrons with anisotropic distributions in solar flares
}

\section{Hard X-rays bremsstrahlung emission}

\author{
V. V. Zharkova ${ }^{1}$, A. A. Kuznetsov ${ }^{2}$, and T. V. Siversky ${ }^{1}$ \\ 1 Department of Computing and Mathematics, University of Bradford, Bradford BD7 1DP, UK \\ e-mail: [v.v.zharkova; t.siverskyi]@brad.ac.uk \\ 2 Institute of Solar-Terrestrial Physics, Irkutsk, Lermontov str. 126a, 664033, Russia \\ e-mail: a_kuzn@iszf.irk.ru
}

Received 8 December 2008 / Accepted 12 November 2009

\begin{abstract}
Aims. The paper aims are to simulate steady-state distributions of electrons beams precipitating in collisional and Ohmic losses with pitch angle anisotropy into a flaring atmosphere with converging magnetic field and to apply these to the interpretation of HXR photon spectra, directivity and polarization observed for different photon energies and flare positions on the solar disk.

Methods. Summary approximation method is applied to a time-dependent Fokker-Planck equation by splitting the temporal derivative equally between the derivatives in depth, energy and pitch angles and finding the solutions in forward and backward directions for each variable.

Results. For softer beams, there is a noticeable flattening of the photon spectra at lower energies caused by the self-induced electric field that increases for larger viewing angles. For the models with an electric field, the HXR emission with lower energies (30 keV) becomes directed mainly upwards at upper atmospheric levels owing to the increased number of particles moving upwards, while in deeper layers it again becomes directed downwards. The polarization maximum shifts to higher energies with every precipitation depth approaching $25 \mathrm{keV}$ for the models with pure collisions and $100 \mathrm{keV}$ for the models with return currents. At deeper layers, the polarization decreases because of the isotropization of electrons by collisions. The maximum polarization is observed at the viewing angle of $90^{\circ}$, becoming shifted to lower angles for softer beams. The integrated polarization and directivity shows a dependence on a magnetic field convergence for harder beams, while for softer beams the directivity is strongly affected by the self-induced electric field changing from a downward motion to an upward one at upper atmospheric depths.

Conclusions. The proposed precipitation model for an electron beam with wider pitch angle dispersion of 0.2 taking into account collisional and Ohmic losses allowed us to fit the double power law HXR photon spectra with a spectrum flattening at lower energies observed in the flares of 20 and 23 July 2002. The observed directivity of HXR photons of $20 \mathrm{keV}$ derived for a large number of flares located from the disk center to limb is also reproduced well by the theoretical directivity calculated for an electron beam with a very narrow pitch angle dispersion of 0.02 . The simulated polarization of this narrowly-directed electron beam fits up to $90 \%$ of all the available polarimetric observations carried out at various locations across the solar disk.
\end{abstract}

Key words. Sun: flares - Sun: X-rays, gamma rays

\section{Introduction}

RHESSI observations have allowed us to enhance in the quantitative interpretation of hard X-ray emission (Lin et al. 2003; Holman et al. 2003; Brown et al. 2006; Krucker et al. 2008; Holman et al. 2010) by providing not only light curves but the locations and shapes of hard X-ray sources on the solar disk, in addition to photon and electron spectra and their evolution during a flare. The observations show that flare events sometimes comprise one loop with two footpoints and one coronal source (Masuda et al. 1994; Sui et al. 2002) or several sets of loops with a number of footpoints and coronal sources (Battaglia \& Benz 2006; Krucker et al. 2008). The analyses of observations of solar flares by TRACE and RHESSI found that the areas of flaring loops decrease and, thus, their magnetic fields increase with depth of the solar atmosphere (Kontar et al. 2008).

A very close temporal correlation is detected between the lightcurves across the full electromagnetic range: HXR, microwave (MW), ultraviolet (UV), and optical emission assuming some common agents which deliver the energy to different atmospheric levels within a timescale shorter than $1 \mathrm{~s}$ (see for example, Fletcher et al. 2010; Holman et al. 2010; White et al. 2010). Although the spatial configurations of flaring sites in HXR and MW emission are not always similar, furthermore, they are very different in optical emission. Assuming that the HXR and MW emission is caused by the same population of electrons, these spatial differences may be indicative of different transport scenarios for high-energy particles precipitating into loop footpoints and different shapes of the flaring atmospheres where this emission occur (Bastian et al. 1998; Kundu et al. 2001a,b; Vilmer et al. 2002; Willson \& Holman 2003; Kundu et al. 2004; Aschwanden 2005)

The mechanisms of transport affecting the HXR and MW emission substantially differ: MW radiation is related to gyro-synchrotron emission of high-energy electrons with energies from few tens keV (Kundu et al. 2001a,b) up to several MeV 
(Bastian 1999; Kundu et al. 2004), while HXR radiation is often produced by electrons of much lower energy from 10 to $300 \mathrm{keV}$ (see for example, Lin et al. 2003; Holman et al. 2003). Although, occasionally, the accelerated electrons can reach energies of a few hundred $\mathrm{MeV}$ as observed for the flare of 28 October 2003 (Kuznetsov et al. 2006).

It is now well accepted that the agents delivering the energy required to account for the observed features in HXR, MW, and other emission are sub-relativistic charged particles (beams of electrons (Brown 1971; Brown et al. 2006), possibly, mixed with protons (Simnett 1995) accelerated somewhere in the corona. These particle precipitate downwards to the lower solar atmosphere, while depositing their energy into the ambient plasma. Obviously, the diagnostics of this precipitation from different types of emission can provide a valuable insight into the complicated processes, in which these particles deposit their energy at various depths of flaring loops. The variety of observations has led to the development of different models of particle acceleration and transport for different types of flaring events (see the reviews by Zharkova et al. 2010; Holman et al. 2010, in the forthcoming RHESSI book), which can account for the observed patterns in high energy emission.

These include double power-law elbow-type spectra of HXR photon emission deduced with the RHESSI payload from powerful flares with flattening towards lower energies (Holman et al. 2003), confirming earlier findings by the SMM observations (Benz 1977; Brown \& Loran 1985) and the soft-hardsoft temporal profile of photon spectral indices below the energy of $35 \mathrm{keV}$ (Grigis \& Benz 2004) during the duration of the same flare. The spectral flattening at lower photon energies was first interpreted by an increase of the lower cutoff energy of beam electrons (Sui et al. 2005) that was later overturned by Sui et al. (2007) in favor of the Ohmic losses caused by the self-induced electric field of precipitating electrons (Zharkova \& Gordovskyy 2006). In this case, the beam electrons producing HXR emission have different initial energy fluxes and, thus, different Ohmic losses: the higher the flux the greater the losses leading to larger spectral flattening in photon spectrum at lower energies (Zharkova \& Gordovskyy 2006). Because of these dependences, the soft-hard-soft patterns in the temporal profile of HXR photon spectra (Krucker et al. 2008) can be easily reproduced by a triangle temporal profile (low-high-low) of the beam energy flux during flaring events (Zharkova \& Gordovskyy 2006).

Another pattern that can be derived from observed MW and HXR emission is related to pitch-angle distributions of the beam electrons producing this emission and to the locations of flares on the solar disk. This pitch angle anisotropy of precipitating particles can be detected by polarimetric observations in flares located between close to the solar center and the limb. These observations are rather scattered since they were carried out over many years in different energy bands: e.g., $15 \mathrm{keV}$ in the early rocket observations by Tindo et al. (1970, 1972a,b), 16-21 keV later by Tramiel et al. (1984), 100-350 keV in the RHESSI observations Suarez-Garcia et al. (2006), and at 200-400 keV in the ACT observations by Boggs et al. (2006).

The polarimetric observations are indicative of the high variability of the HXR polarization degrees in different events: from a few percent (Tramiel et al. 1984) up to $40 \%$ (Tindo et al. 1976; Suarez-Garcia et al. 2006), or even above $50 \%$ as observed for the flare of 23 July 2002 (McConnell et al. 2003) or $75 \%$ as observed by the CORONAS payload (Zhitnik et al. 2006). The bulk of observations also detect a noticeable increase of the HXR polarization and directivity in flares located closer to the limb, although some deviations from this trend have been recently observed by RHESSI (Suarez-Garcia et al. 2006). The similar increase in the HXR directivity at $20-150 \mathrm{keV}$ in flares located towards the limb is reported by Kašparová et al. (2007).

The theory describing generation of bremsstrahlung hard $\mathrm{X}$-ray emission has significantly progressed due to improvements in the mechanisms for emitting this radiation: from pure Coulomb collisions (Brown 1971; Brown et al. 2006; Kontar \& Brown 2006) to collisions and converging magnetic fields (Leach \& Petrosian 1981; McClements 1992), or to combined collisional and Ohmic energy losses in the electric field induced by precipitating electrons (Zharkova et al. 1995; Zharkova \& Gordovskyy 2005, 2006). Further progress was achieved by considering relativistic bremsstrahlung cross-sections (Kontar et al. 2006) and taking into account various aspects of the photospheric albedo effects, while deriving mean electron spectra from the observed bremsstrahlung photon spectra (Kontar et al. 2006).

However, in spite of a large number of simulations for interpreting HXR or MW emission (see for example, reviews by Krucker et al. 2008; Holman et al. 2010; White et al. 2010, and references therein), no simultaneous simulations have been developed yet that account for these two types of emission with the same distributions of electrons. If one assumes that HXR and MW emissions are generated by different energy loss mechanisms of the same beam electrons, a rather challenging task is to account for the temporal variations of their depth distributions and the total areas of a given intensity covered in each precipitation site. This imposes strict limitations on the proposed models of electron precipitation that require us to correctly account for electron energy losses, such as Coulomb collisions, Ohmic losses and anisotropic scattering and changes in the electron pitch angles caused by a magnetic field that converges with depth.

The motivation of the present paper is to simulate electron distributions during their precipitation into a flaring atmosphere by taking into account all possible energy losses, and to produce HXR spectra and polarization matching those inferred from observations. This paper deals with the precipitation mechanisms of electrons with a wide range of energies from $10 \mathrm{keV}$ to $1.2 \mathrm{MeV}$ and their effect on HXR emission and polarization. This approach significantly extends the steady solutions for electron distribution functions found by Zharkova \& Gordovskyy (2005), considering a magnetic field convergence, in addition to self-induced electric field, to much higher energies of electrons: from $384 \mathrm{keV}$ to $1.2 \mathrm{MeV}$, required to account correctly for a magnetic mirroring, and to the inclusion of a pitch-angle diffusion term, in order to account for the particle and photon anisotropy, similar to the approach by Zharkova et al. (1995). In addition, in contrast to the angle-independent cross-section (averaged over all pitch angles) used by Zharkova \& Gordovskyy (2005), the simulations of HXR bremsstrahlung emission are performed by using the relativistic angle-dependent photon cross-sections that are similar to those of Bai \& Ramaty (1978).

The kinetic problem of precipitation of high-energy electrons from the source within the corona into a converging coronal loop with pitch-angle diffusion and energy losses in the induced electric field is described in Sect. 2. The depth, energy and pitch-angular variations in electron distribution functions of the injected and returning beams, resulting hard X-ray photon spectra and their polarization are discussed in Sect. 3 with conclusions being drawn in Sect. 4. 


\section{Electron precipitation: Fokker-Planck approach}

\subsection{The basic equations}

We consider the time-dependent Fokker-Planck approach to the precipitation of high-energy electrons injected into a cold hydrogen plasma confined in a converging magnetic field structure. However, since high-density electron beams precipitating into a flaring atmosphere are expected to be rather dense, so they can carry a strong electric field. This electric field forms a return current from both the ambient plasma (Knight \& Sturrock 1977; van den Oord 1990) and precipitating electrons returning to the source in the corona (Emslie 1980; Zharkova et al. 1995). Therefore, to accurately consider the beam electron kinetics during precipitation, one needs to include this induced electric field because it increases pitch-angle anisotropy of precipitating beam electrons as described by Diakonov \& Somov (1988); McClements (1992); Zharkova \& Gordovskyy (2005) and Siversky \& Zharkova (2009).

Thus, one needs to solve simultaneously the kinetic FokkerPlanck equation for beam electron precipitation and Ohm's law for the electric field carried by the beams themselves at every depth. In the kinetic equation, we neglect the interaction of beam electrons with waves occurring in the ambient plasma since the energy exchange between electrons and plasma waves does not significantly affect the energy balance of fast electrons (Benz 2002; Aschwanden 2005). In the equation for the electric field we assume that the electric field induced by beam electrons is fully compensated by the electrons of the ambient plasma.

The Fokker-Planck, or Landau, equation, which describes the variations of distribution function $f$ of beam electron, can be written in the form (Landau 1937; Siversky \& Zharkova 2009)

$$
\begin{aligned}
& \frac{\partial f}{\partial t}+v \cos \alpha \frac{\partial f}{\partial z}-e \mathcal{E} v \cos \alpha \frac{\partial f}{\partial E}-\frac{e \mathcal{E} \sin ^{2} \alpha}{m_{\mathrm{e}} v} \frac{\partial f}{\partial \cos \alpha}= \\
& \left(\frac{\partial f}{\partial t}\right)_{\text {coll }}+\left(\frac{\partial f}{\partial t}\right)_{\text {magn }},
\end{aligned}
$$

where $E$ and $\alpha$ are the electron energy and pitch angle, respectively, $v$ is the electron speed, and $e$ and $m_{\mathrm{e}}$ are the electron charge and mass, respectively. The variations of distribution function with time $(t)$ are described by the first term on the lefthand side, with depth $(z)$ by the second term and with energy and pitch angle from Ohmic losses in the self-induced electric field $\mathcal{E}$ by the third and the fourth terms. The particle energy and pitchangle diffusion caused by energy losses in collisions with the ambient plasma particles (collisional integral) and in a converging magnetic field are described by the two terms on the righthand side, $(\partial f / \partial t)_{\text {coll }}$ and $(\partial f / \partial t)_{\text {magn }}$, respectively. The distribution function is normalized by the initial beam density on the top boundary (Siversky \& Zharkova 2009), which differs from those in Zharkova \& Gordovskyy (2005) where the normalization was by the initial energy flux of beam electrons.

\subsubsection{The initial and boundary conditions}

We assume that there are no beam electrons inside the atmosphere at the moment of injection, e.g., $f(E, \mu, z, 0)=0$.
The distribution function on the top boundary where $z=z_{\min }$ is defined by the formula

$$
\left.f(E, \mu, t)\right|_{z=z_{\min }}=\left\{\begin{array}{l}
A E^{-\gamma-0.5} \exp \left[-\frac{(\mu-1)^{2}}{\Delta \mu^{2}}\right] U(t), \\
\text { for } E_{\min } \leq E \leq E_{\max }, \mu>0, t \geq 0, \\
0, \text { elsewhere, }
\end{array}\right.
$$

which assumes the power-law energy distribution in the energy range from $E_{\min }$ to $E_{\max }$ with a spectral index $\gamma$ and the normal pitch angle distribution with respect to the pitch-angle cosine $\mu(\mu=\cos \alpha)$ with the half-width dispersion $\Delta \mu$ (Siversky \& Zharkova 2009).

Let us consider an electron beam injected with a power-law dependence in energy and a narrow normal distribution in pitch angle with the angle dispersion $(\Delta \mu \ll 1)$ centered on $\alpha=0$ (or $\mu=1$ ). The coefficient $A$ is inferred from the normalization condition for a distribution function on a beam density on the top boundary (Siversky \& Zharkova 2009). The additional index of 0.5 is added to index $\gamma$ in the the energy dependence, in order to account for the spectral index $\gamma$ being referred to the electron flux $\left(\sim v f=E^{0.5} f\right)$ and not to the electron density, by which $f$ is normalized. $U(t)$ defines a temporal profile of the electron beam injection, which denotes the initial beam flux variations during a required time interval, accepted in our simulations to be equal to unity (a steady injection). The boundary conditions are the same as those described by Siversky \& Zharkova (2009).

\subsubsection{Energy losses}

The Fokker-Planck equation presented here in Eq. (1) takes into account the energy loss factors such as:

1. particle collisions and anisotropic pitch angle scattering (the term $\left.(\partial f / \partial t)_{\text {coll }}\right)$;

2. return current (caused by the self-induced electric field $\mathcal{E}$ );

3. inhomogeneity (convergence) of a magnetic field (the term $\left.(\partial f / \partial t)_{\text {magn }}\right)$.

The collisional integral is taken in the linearized form suggested by (Diakonov \& Somov 1988)

$$
\begin{aligned}
\left(\frac{\partial f}{\partial t}\right)_{\text {coll }}= & \frac{1}{v^{2}} \frac{\partial}{\partial v}\left[v^{2} v(E)\left(\frac{k_{\mathrm{B}} T_{\mathrm{e}}}{m_{\mathrm{e}}} \frac{\partial f}{\partial v}+v f\right)\right] \\
& +v(E) \frac{\partial}{\partial \cos \alpha}\left(\sin ^{2} \alpha \frac{\partial f}{\partial \cos \alpha}\right),
\end{aligned}
$$

where $T_{\mathrm{e}}$ is the electron temperature of the ambient plasma, $k_{\mathrm{B}}$ is the Boltzmann constant, and $v(E)$ is the frequency of collisions (Emslie 1978)

$$
v(E)=\frac{k}{\sqrt{2 m_{\mathrm{e}}}} \pi n \lambda e^{4} E^{-3 / 2},
$$

where $\lambda$ is the Coulomb logarithm for electrons, $n$ is the ambient plasma density, and the parameter $k$ defines beam electrons scattering on the ambient particles Emslie (1978).

The magnetic field convergence is considered in the following form (McClements 1992)

$$
\left(\frac{\partial f}{\partial t}\right)_{\text {magn }}=\frac{1}{2} v \sin ^{2} \alpha \frac{\partial \ln B(z)}{\partial z} \frac{\partial f}{\partial \cos \alpha},
$$

where $B(z)$ is the magnetic field at a given depth $z$. 
Instead of the physical coordinate $z$, it is convenient to express the distance passed by particles in terms of the column density $\xi$, e.g., $\xi(z)=\int^{z} n(l) \mathrm{d} l$. A magnetic field convergence $B$ can be then described by the hybrid model from Siversky \& Zharkova (2009)

$\frac{\partial \ln B}{\partial \xi}=\frac{1}{n} \frac{\partial \ln B}{\partial z}= \begin{cases}\frac{1}{\xi_{\mathrm{c}}} \ln \frac{B_{\mathrm{c}}}{B_{0}}, & \xi<\xi_{\mathrm{c}}, \\ 0, & \xi \geq \xi_{\mathrm{c}}\end{cases}$

which defines the magnetic field to increase by the factor $B_{\mathrm{c}} / B_{0}$ in the depth interval between the electron injection point $(\xi=0)$ and the transition region $\left(\xi=\xi_{\mathrm{c}}\right)$ (Leach \& Petrosian 1983) and to be a constant in deeper layers.

\subsubsection{Self-induced electric field}

The electric current carried by beam electrons can be determined as

$j(\xi)=e \int_{E_{\min }}^{E_{\max }} v(E) \mathrm{d} E \int_{-1}^{1} f(\xi, E, \mu) \mu \mathrm{d} \mu$.

At each precipitation depths this electric field causes preferential scattering of beam electrons towards negative $\mu$ and the formation of returning beam electrons $(\mu<0)$ with their own electric field directed in the opposite direction. At every precipitation depth, the electric field carried by returning electrons $(\mu<0)$ reduces the electric field induced by the precipitating electrons $(\mu>0)$. This reduces the resulting electric field produced by beam electrons causing a return current from the ambient electrons at a given depth (van den Oord 1990; Siversky \& Zharkova 2009).

Therefore, the electric field carried by the beams themselves at every depth, which is used in the Fokker-Plank equation (Eq. (1)) can be written as follows

$\mathcal{E}=\frac{j(\xi)}{\sigma(\xi)}=\frac{e}{\sigma(\xi)} \int_{E_{\min }}^{E_{\max }} v(E) \mathrm{d} E \int_{-1}^{1} f(\xi, E, \mu) \mu \mathrm{d} \mu$,

where $\sigma(\xi)$ is the classical conductivity of the ambient plasma at a given precipitation depth defined as (Spicer 1977)

$\frac{1}{\sigma}=\frac{7.28 \times 10^{-8} X}{T_{\mathrm{e}}^{1.5}} \ln \left(\frac{3}{2 e^{3}} \frac{k_{\mathrm{B}}^{3} T_{\mathrm{e}}^{3}}{\pi n}\right)+\frac{7.6 \times 10^{-18}(1-X)}{X} T_{\mathrm{e}}^{0.5}$,

where $X$ is the ionization degree.

\subsubsection{Method of solution and accepted parameters}

The set of Eqs. (1) and (4) define the electron beam precipitation into a flaring atmosphere. Unlike the stationary solutions found by Zharkova \& Gordovskyy (2005) (e.g., excluding the time derivative), we solve the full time-dependent Fokker-Planck (FP) equation above. In the solution we also include the term with pitch angle diffusion (a second derivative by $\mu$ ) and magnetic field convergence and yet exclude the diffusion in energy (the first term in the collisional integral). For the FP solutions, we use the summary approximation method (Samarskii 2001) by applying the iteration method by dividing the temporal difference evenly between three other variables (depth, energy and pitch angles); this method already has proven to give reliable time-dependent solutions for both short impulses and steady injection (Zharkova et al. 1995; Siversky \& Zharkova 2009).

The converging magnetic field acts as a magnetic mirror that can increase the number of electrons moving upwards. We determine the magnetic convergence parameter, $\alpha_{\mathrm{B}}$ by selecting a characteristic column depth that defines the scale of the magnetic field change in the selected flaring atmospheres. By comparing the 4 models currently considered in the literature (Siversky \& Zharkova 2009), in the current paper, we adopt the convergence model with a scale column depth of $\xi=10^{20} \mathrm{~cm}^{-2}$ which closely corresponds to values deduced from observations (Kontar et al. 2008).

In the current paper we consider relatively long beam injection times of a few seconds, and, therefore, seek the solutions of the quasi-stationary Eq. (1), i.e. those obtained for the time-dependent equation after the time interval when the stationary injection is established and fully compensated by returning electrons. This steady state is normally achieved for a beam with $\gamma=3$ and an initial energy flux $F=10^{10} \mathrm{erg} \mathrm{cm}^{-2} \mathrm{~s}^{-1}$ at about $0.07 \mathrm{~s}$ after the onset of beam injection (Siversky \& Zharkova 2009).

\subsection{Hard $X$-ray bremsstrahlung emission and polarization}

The parameters of X-ray emission from a homogeneous source in the solar corona (observed at the Earth) are determined by the equation (Elwert \& Haug 1970; Brown 1972; Leach \& Petrosian 1983)

$$
\begin{aligned}
{\left[\begin{array}{l}
I \\
Q
\end{array}\right](h v, \theta)=} & \frac{n_{\mathrm{i}} V}{2 \pi \mathcal{R}^{2}} \int_{h v}^{\infty} v(E) \mathrm{d} E \int_{-1}^{1} f(E, \mu) \mathrm{d} \mu \\
& \times \int_{0}^{2 \pi}\left[\begin{array}{c}
\sigma_{I} \\
\sigma_{Q}
\end{array}\right](h v, \theta, E, \mu, \varphi) \mathrm{d} \varphi,
\end{aligned}
$$

where $I$ and $Q$ are the Stokes parameters of bremsstrahlung radiation, $h v$ and $\theta$ are the energy and the propagation angle (with respect to the magnetic field, i.e., pitch angle) of the X-ray photons, $n_{\mathrm{i}}=n$ is the concentration of the background ions, $V$ is the source volume, $\mathcal{R}$ is the astronomical unit, the parameters $\sigma_{I, Q}$ describe the bremsstrahlung probability (see Appendix A), and $\varphi$ is the electron azimuthal angle. The factor $2 \pi$ is related to the normalization condition, since the distribution function of electrons is assumed to be azimuthally symmetrical. In this case, another Stokes parameter $(U)$ equals zero (Bai \& Ramaty 1978; Leach \& Petrosian 1983).

We assume that the X-ray source can be represented by a cylindrical magnetic tube in which the magnetic field is aligned along the tube axis. All the parameters of the plasma, magnetic field, and accelerated particles depend only on the coordinate $z$ along the tube. In this case, the total (integrated over all layers of the source) emission is determined by the equation

$$
\begin{aligned}
{\left[\begin{array}{l}
I \\
Q
\end{array}\right](h v, \theta)=} & \frac{S}{2 \pi \mathcal{R}^{2}} \int_{0}^{\xi_{\max }} \mathrm{d} \xi \int_{h v}^{\infty} v(E) \mathrm{d} E \int_{-1}^{1} f(\xi, E, \mu) \mathrm{d} \mu \\
& \times \int_{0}^{2 \pi}\left[\begin{array}{c}
\sigma_{I} \\
\sigma_{Q}
\end{array}\right](h v, \theta, E, \mu, \varphi) \mathrm{d} \varphi
\end{aligned}
$$


where $S$ is the cross-section area of a fluxtube. In a converging magnetic field, the cross-sectional area varies (due to the conservation of the magnetic flux) as $S(\xi) \sim B^{-1}(\xi)$, and the concentration of the accelerated particles varies as $n_{\mathrm{e}}(\xi) \sim B(\xi)$. Thus, those variations compensate each other and do not need to be considered.

The degree of the linear polarization of the emission is defined by

$\eta=\frac{Q}{I}$

and the angular distribution of the emission can be characterized by the so-called directivity parameter (Brown 1972; Leach \& Petrosian 1983)

$D(\theta)=\frac{I(\theta)}{\langle I\rangle}$,

where $\langle I\rangle$ is the emission intensity averaged over all propagation angles. The three-dimensional integrals over the components of the electron velocity in (6-7) were calculated using Monte-Carlo method.

\section{Results and discussion}

\subsection{Dynamics of electron beams}

In all calculations, anisotropic scattering is considered, i.e, a dependence of electron distributions on the pitch angle at scattering. A few precipitation models were used to investigate the combined effects of different energy losses: a) C - only collisions with charged particles and neutrals, b) $\mathrm{CE}$ - collisions and self-induced electric field, c) CB - collisions and converging magnetic field, and d) CEB - collisions, a self-induced electric field, and a converging magnetic field. In the simulations, we used the following parameters: initial energy flux of the accelerated electrons $F=10^{10} \mathrm{erg} \mathrm{cm}^{-2} \mathrm{~s}^{-1}$, electron energy range defined by $E_{\min }=12 \mathrm{keV}, E_{\max }=1200 \mathrm{keV}$, initial pitch-angle dispersion of the electrons $\Delta \mu=0.2$, and a transition region boundary at $\xi_{\mathrm{c}}=10^{20} \mathrm{~cm}^{-2}$. The initial power-law index of the accelerated electrons was taken to be either $\gamma=3$ or $\gamma=7$. The convergence factor $B_{\mathrm{c}} / B_{0}$ was equal to $B_{\mathrm{c}} / B_{0}=3$ and the characteristic column depth to $2 \times 10^{20} \mathrm{~cm}^{-2}$ (for details of the selection rule, see Siversky \& Zharkova 2009).

Examples of the distribution functions of electron for a few column depths are shown in Figs. 1-3: a) for the column density of $\xi=10^{18} \mathrm{~cm}^{-2}$ (Fig. 1), b) for the column density of $\xi=$ $10^{20} \mathrm{~cm}^{-2}$ (Fig. 2), and c) for the column density $\xi=10^{22} \mathrm{~cm}^{-2}$ (Fig. 3). One can see that both the self-induced electric and the converging magnetic fields produce a particle stream that propagates upwards, or returns back to the coronal source, from which it is injected. This stream significantly exceeds a random stream of particles scattered isotropically and becomes comparable to the stream of the particles propagating downwards, or precipitating particles.

The self-induced electric field of the beam produces a stream of returning beam electrons (Figs. 1a and 1d) that compensates for the charge of precipitating electrons from Eq. (4). The selfinduced electric field at all depths comprises an electric field of precipitating electrons minus the electric field of the beam electrons, which changed their propagation direction at the depths above to the negative $\mu$. This electric field corresponds to one that is then compensated for by the ambient electrons known as a return current (van den Oord 1990). We note that for electron beams with extended energy ranges up to $1.2 \mathrm{MeV}$, the effect of a self-induced electric field remains more important at upper atmospheric depths for beams with softer energy spectra (compare the left graphs (a, d) in Figs. 1-3), confirming the conclusions of Zharkova \& Gordovskyy $(2005,2006)$. One can also see that the self-induced electric field is able to accelerate the returning beam particles to rather high energies (up to 50-100 keV), while their number drops to zero at higher energies.

The distributions of returning electrons also depart from power laws resembling quasi-thermal distributions. The variations in power-law index $\gamma$ in the energy distributions of injected particles lead to a number of precipitating electrons that decreases with energy much faster for $\gamma=7$ than for $\gamma=3$. As a result, for $\gamma=7$, at some atmospheric depths (Fig. 1), the number of returning particles with energies below $100 \mathrm{keV}$ can even exceed the number of precipitating particles with the same energies, which dramatically affects the properties of the X-ray emission, as discussed in Sect. 3.2.

On the other hand, a converging magnetic field produces the well-known loss-cone distributions by reflecting the precipitating electrons being scattered to pitch angles within the losscone defined by the magnetic convergence factor (Figs. (1-3)b and (1-3)e). The energy spectrum of the returning particles (reflected by a converging magnetic field) does not differ significantly from the initial energy spectrum of precipitating particles. However, the effect of magnetic mirroring is more significant for higher energy electrons than other energy losses (collisions or Ohmic losses) (compare Figs. 3a, d and c, f with Fig. 1a, d and $\mathrm{c}, \mathrm{f}$, respectively). This is because energy losses depend inversely on the particle energies, thus becoming with depth (and energy loss) much smaller than the energy losses in magnetic mirroring (convergence). In real events (e.g., solar flares), all the energy loss factors operate simultaneously, producing the beam electron distributions in energy and pitch angles perhaps similar to those shown in Figs. $(1-3) \mathrm{c}$ and $(1-3) \mathrm{f}$.

At depths of $\xi \simeq 10^{20} \mathrm{~cm}^{-2}$ where the bulk of electrons lose their energy, the magnetic field convergence and collisions govern the remaining electron distributions (Fig. 2). The electrons are distributed almost isotropically at all energies (due to collisional scattering) and the converging magnetic field makes the isotropisation process faster. In deeper layers (Fig. 3), the number of particles decreases rapidly with depth because of collisions. Since the collisional losses are more significant for lowenergy electrons, the "humped" distribution (with a maximum at about $100 \mathrm{keV}$ ) is formed.

Figure 4 shows the density variations of energetic electrons, where the distance from the injection point is expressed as a column density. If the self-induced electric field is not taken into account (see the panels labeled $\mathrm{C}$ and $\mathrm{C}+\mathrm{B}$ ), then the electric currents directed upwards and downwards do not compensate each other because only some of the precipitating electrons are scattered to negative pitch angle cosines, as described by Eq. (4) for the self-induced electric field. The beam densities of returning electrons depend on the energy loss mechanisms of precipitating electrons. We note that in the corona (at $\xi<\xi_{10} \simeq 2 \times 10^{19} \mathrm{~cm}^{-2}$ ), the electron energy losses due to collisions are insignificant, while Ohmic losses can play a dominant role (compare the magnitudes of collisional and electric stopping depths defined in Table 1 of Zharkova \& Gordovskyy 2006). Thus, the number of returning particles (integrated over all energies) is always slightly less than that of the total precipitating particles.

At every precipitation depth in the corona, there is a substantial increase in the numbers of particles that propagate upwards (as shown in the panels labeled $\mathrm{C}+\mathrm{E}$ and $\mathrm{C}+\mathrm{E}+\mathrm{B}$ in Figs. 1-3), 

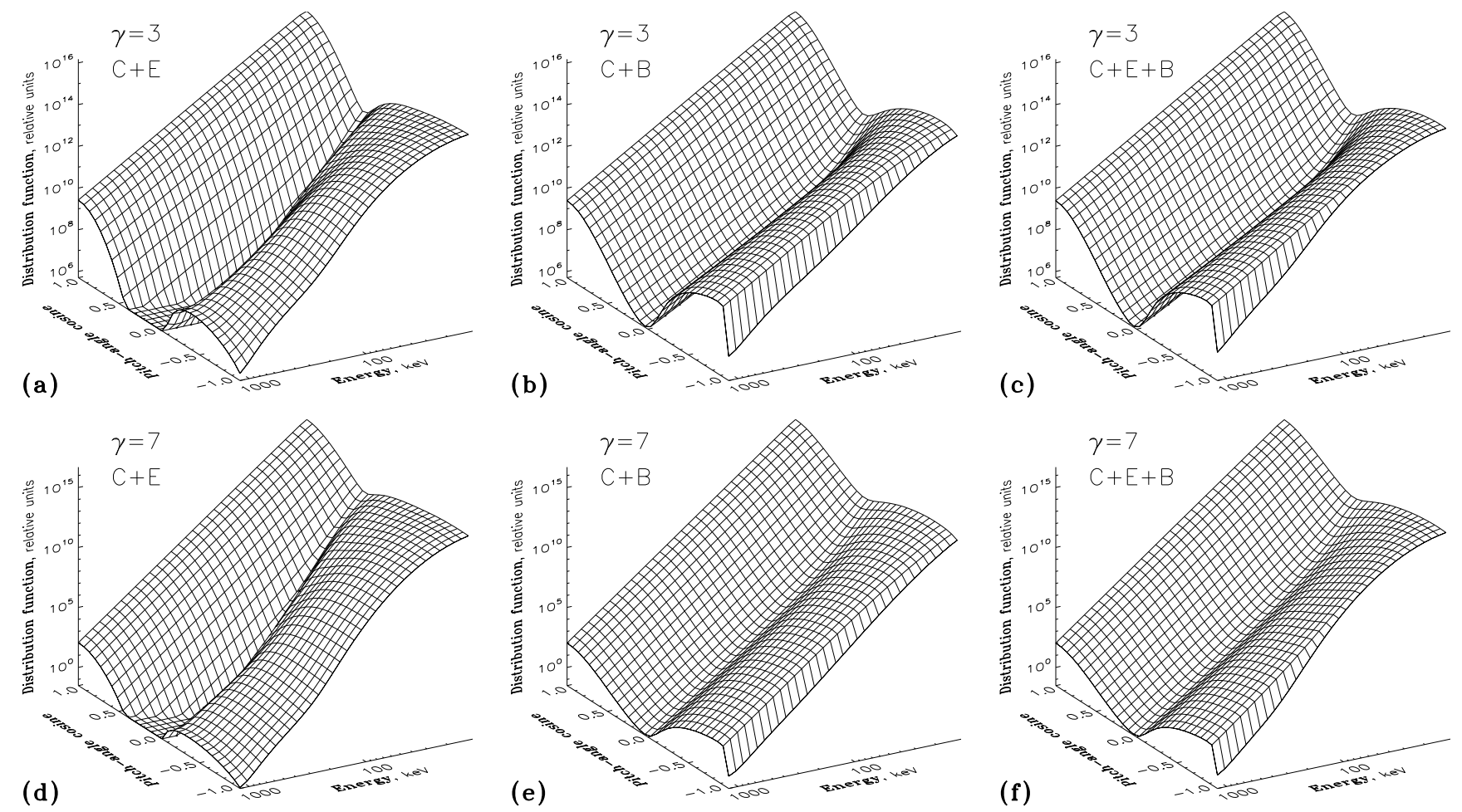

Fig. 1. Electron distribution functions (in the $E-\mu$ space) obtained by solving the Fokker-Planck equation at the column depth of $\xi=10^{18} \mathrm{~cm}^{-2}$. The initial power-law indices of the accelerated electrons are equal to 3 and 7. The factors taken into account are: collisions (C), self-induced electric field (E), and convergence of the magnetic field (B).
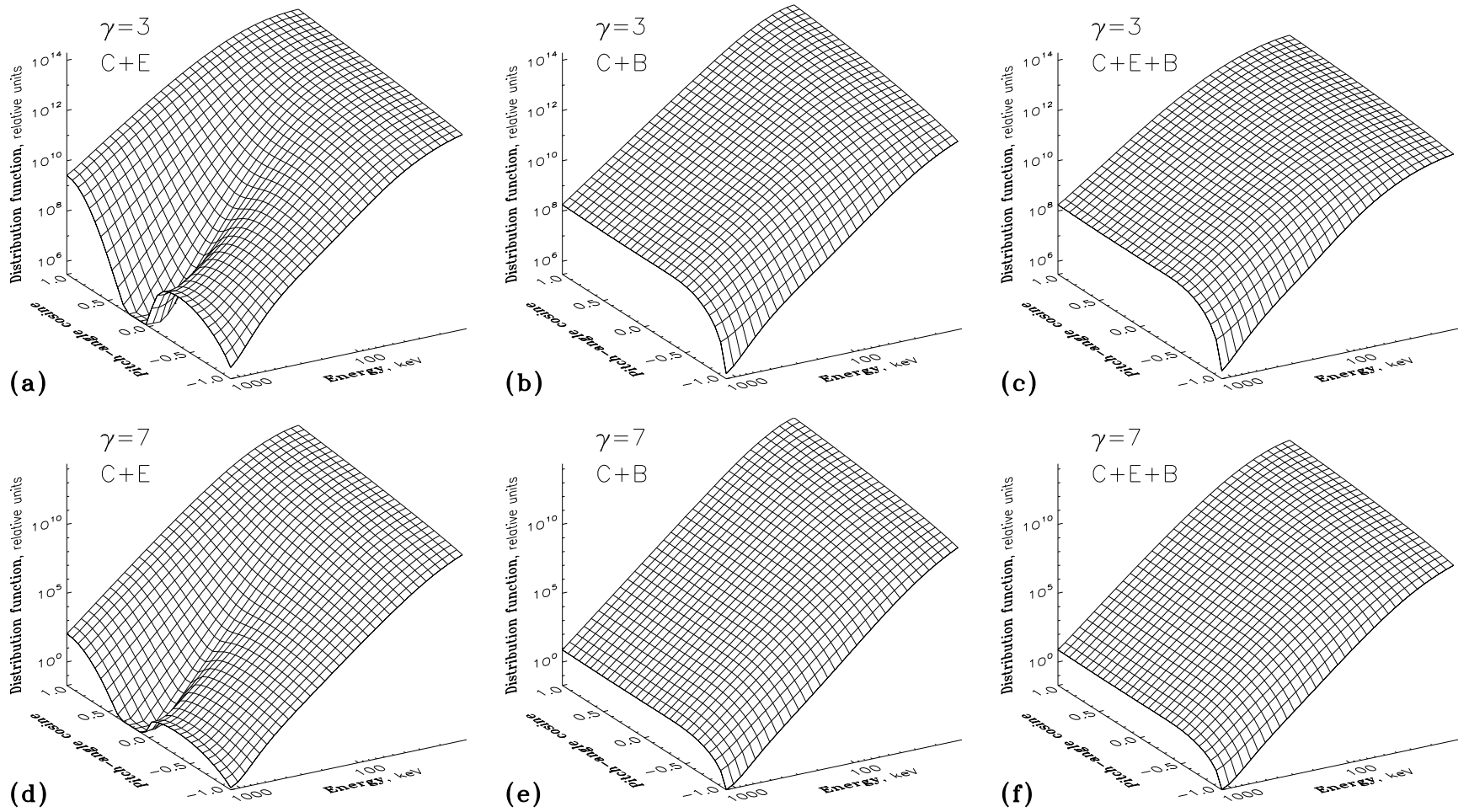

Fig. 2. Electron distribution functions at the column depth of $\xi=10^{20} \mathrm{~cm}^{-2}$. Other simulation parameters are the same as in Fig. 1.

this increase becoming higher for the beams with larger spectral indices (compare Figs. 4a and 4b). In other words, the electric current is not simply proportional to the density of precipitating particles as discussed in Sect. 2 but depends on the spectral characteristics of beam electrons indicated by Eq. (4). Our simulations confirm the well-known fact that electron beams with harder spectra can reach deeper layers of the solar atmosphere (Zharkova \& Kobylinskii 1993; Zharkova \& Gordovskyy 2005; Siversky \& Zharkova 2009). This produces higher densities at deeper atmospheric levels for the electron beams with $\gamma=3$ than for those with $\gamma=7$, while at upper atmospheric levels the densities of returning electrons are higher for the electrons 

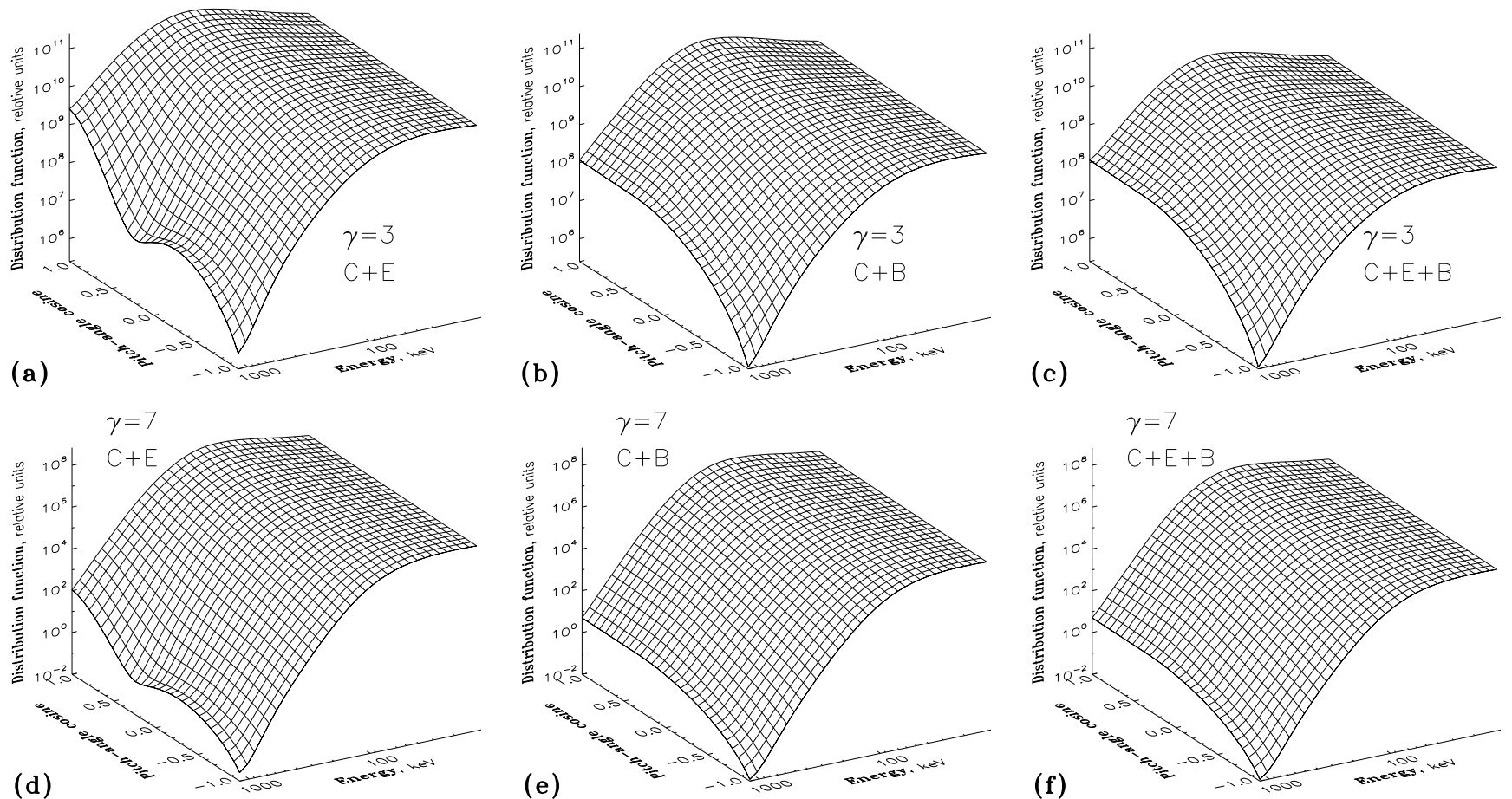

Fig. 3. Electron distribution functions at the column depth of $\xi=10^{22} \mathrm{~cm}^{-2}$. Other simulation parameters are the same as in Fig. 1 .
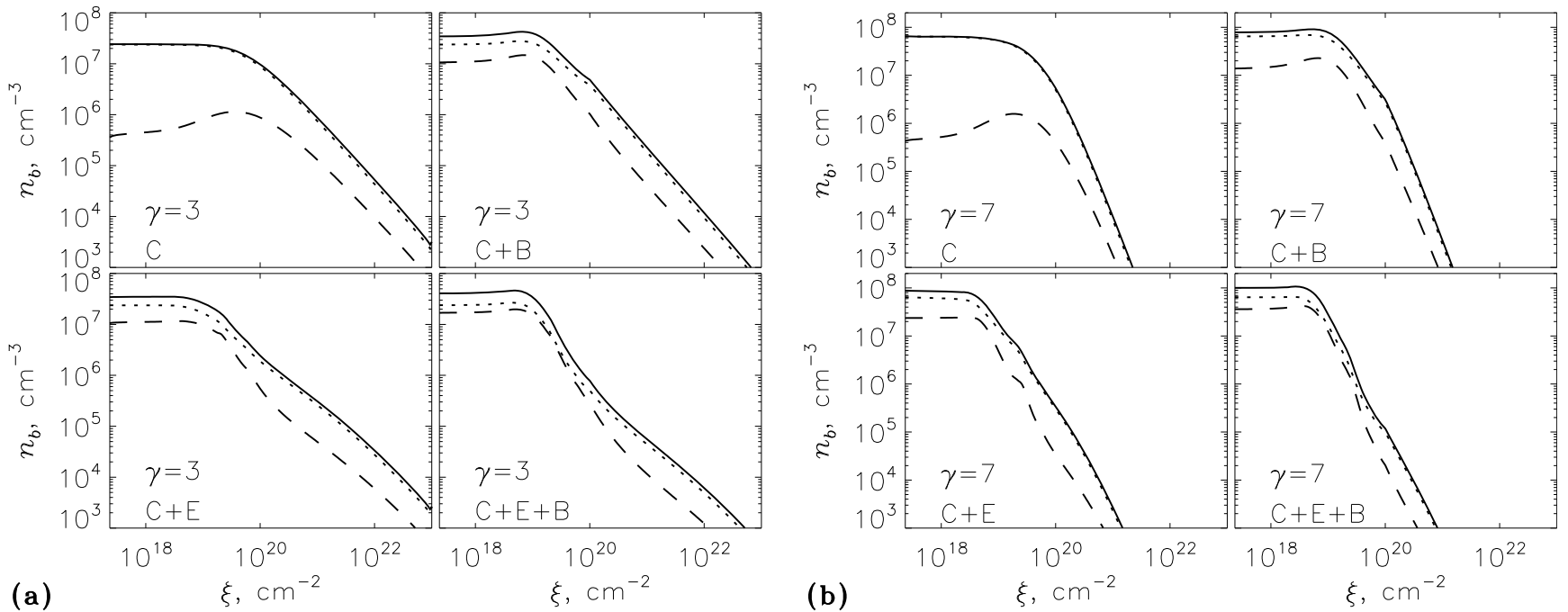

Fig. 4. Dependencies of the density of precipitating electrons on a column density of the ambient plasma. Dotted line: downward propagating particles $(\mu>0)$; dashed line: upward propagating particles $(\mu<0)$; solid line: total concentration (downward + upward). In the different panels, the electron distributions are obtained by taking into account different factors (see Fig. 1).

with $\gamma=7$, reflecting the Ohmic loss dependence of the powerlaw index (Zharkova \& Gordovskyy 2006). The effect of magnetic mirroring for the $\mathrm{C}+\mathrm{E}+\mathrm{B}$ models only enhances the number of returning electrons across the entire energy range as represented by Figs. 1-3.

\subsection{Variations in $H X R$ bremsstrahlung intensity and polarization}

\subsubsection{Depth variations of $H X R$ bremsstrahlung emission}

The hard X-ray bremsstrahlung intensity and polarization produced by electrons injected with power-law spectral indices of $\gamma=3$ (red lines) and $\gamma=7$ (blue lines) are shown in Fig. 5.
The first three columns present the X-ray photon spectra for the different simulation models and propagation directions, at three distances from the electron injection point. We note that the curves for different electron power-law indices are drawn by using a different scaling factor, so the direct comparison of HXR emission caused by these beams with their assigned spectral indices is infeasible. The last column of Fig. 5 shows the polarization of HXR emission calculated for a pitch angle $\theta=90^{\circ}$, where it is the highest.

We first consider the emission from the electron beams with $\gamma=3$ (shown by red lines in Fig. 5). The spectrum of emission from the initial electron beam (at the injection point) with a power-law distribution is shown in Fig. 5a. Hard X-ray emission, especially at higher energies, is emitted preferably in the 
A\&A 512, A8 (2010)
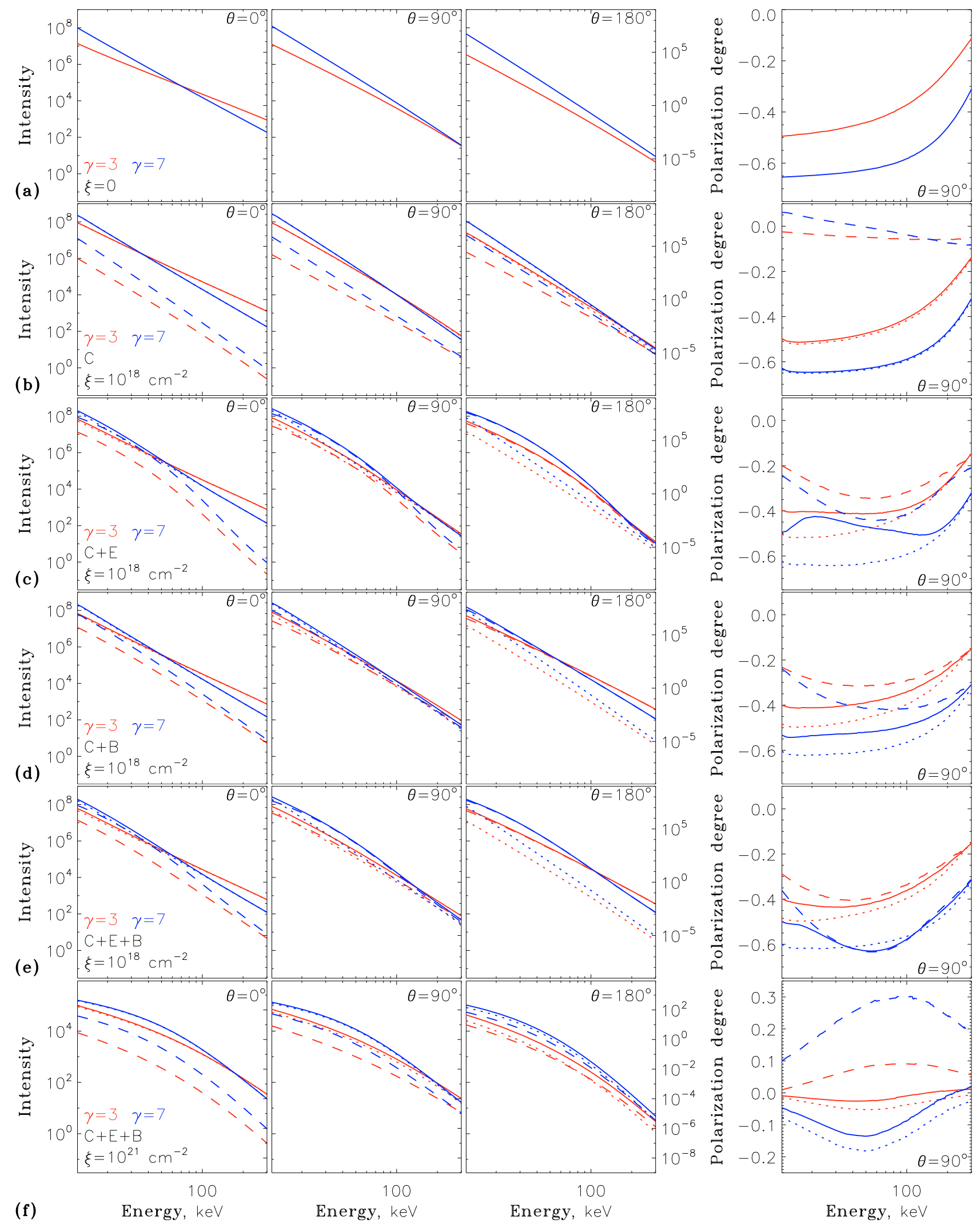

Fig. 5. Intensity (in relative units) and polarization of the hard X-ray emission for the different energies. Initial power-law indices of the accelerated particles are: $\gamma=3$ (red lines) and $\gamma=7$ (blue lines). The intensity profiles for $\gamma=3$ and $\gamma=7$ are indicated by using a different scaling; the intensity values are shown on the left (for $\gamma=3$ ) and on right (for $\gamma=7$ ) margins, respectively. Dotted line: emission of the downward propagating particles (with $\mu>0$ ); dashed line: emission of the upward propagating particles (with $\mu<0$ ); solid line: total emission (downward + upward). The different panels (from a to f) correspond to the different depths $(\xi)$ and the different simulation models (see Fig. 1). 
direction of electron propagation $\left(\theta \simeq 0^{\circ}\right)$. Thus, with increasing viewing, or pitch, angle $\theta$, the emission intensity is expected to decrease, and the spectrum becomes softer, if the bulk of electrons continue to propagate downwards. However, as the scattering intensity of electrons increases with depth, the above conclusion becomes not valid because the electrons emit far more isotropically.

The intensity plots in Figs. 5b-5e illustrate the effects of collisions, a self-induced electric field, or a converging magnetic field on the emission, at some distance from the injection point ( $\xi=10^{18} \mathrm{~cm}^{-2}$ corresponding to the corona). Figure $5 \mathrm{~b}$ illustrates the effect of collisions with pitch-angle scattering on the HXR emission emitted by the downward (dotted line) and upward (dashed line) moving particles. As we established in Sect. 3.1, the number of particles scattered by pure collisions to large angles (when $\mu$ changes sign) remains very small. This explains why the coronal emission from precipitating electrons is an order of magnitude higher than those in the chromosphere (compare rows b and f), while the shape of the energy spectrum does not change much with depth. The emission from returning electrons (dashed line) is much smaller than for the precipitating ones. Emission from returning electrons becomes closer to those from precipitating ones as the viewing angle increases.

Figure $5 \mathrm{c}$ shows the effect of a self-induced electric field and the appearance of the electrons of a return current (moving with $\mu<0$ ), which have a considerable effect on the resulting HXR emission spectra. This effect is strongest at $h v<60 \mathrm{keV}$, but is noticeable at the higher energies as well (up to $300 \mathrm{keV}$ ) for beams with an upper energy cutoff of $1.2 \mathrm{MeV}$. The upward emission $\left(\theta=180^{\circ}\right)$ is generated entirely by electrons of the return current. The emission intensity (especially at energies of about $30-100 \mathrm{keV}$ ) is much higher than for collisions with anisotropic scattering without the return current. The emission spectrum at a depth of $10^{18} \mathrm{~cm}^{-2}$ differs from a power-law, the spectrum apparently flattening at lower energies while keeping its power-law shape at higher energies. This effect was noted previously by Zharkova \& Gordovskyy $(2005,2006)$.

The spectral flattening at low energies is also visible at the viewing angle $\theta=90^{\circ}$, although is much weaker. The contributions of the downward and upward electrons become almost equal. For the downward emission $\left(\theta=0^{\circ}\right)$, the contribution of the downward electrons always far exceeds that of the upward electrons. Nevertheless, even in this case the return current electrons affect the spectrum shape at energies below $50 \mathrm{keV}$.

The effect of a converging magnetic field is illustrated in Fig. 5d. The convergence effect is strongest at $\theta=180^{\circ}$, where emission at higher energies is produced entirely by reflected electrons, and at lower energies $(<30 \mathrm{keV})$ the contribution of the reflected particles dominates. The emission intensity is about two times higher than in the case of pure collisions (Fig. 5b), and the spectrum is harder. For the transversal propagation $\left(\theta=90^{\circ}\right)$, the contributions of the downward and reflected electrons are comparable, and the spectra of emission produced by these electron populations are almost identical. For the downward emission propagation $\left(\theta=0^{\circ}\right)$, the contribution of the reflected electrons into X-ray emission is negligible.

The intensities and polarization calculated for the most realistic case that includes all the energy loss effects, e.g., collisions, return current, and magnetic field convergence, are plotted in Fig. 5e. One can see that two factors that produce the upward electron flux are now combined: the return current electrons increase the upward emission intensity at lower energies $(<100 \mathrm{keV})$, while the reflected (by the converging magnetic field) particles enhance the high-energy tail of the emission spectrum. We note that the converging magnetic field removes the spectral flattening at lower energy as seen in Fig. 5c (for the beam with $\gamma=3$ ). The emission spectra at all viewing directions become similar to that in Fig. 5d. We conclude that for hard electron beams, the influence of the converging magnetic field is stronger than that of the self-induced electric field.

The emission spectra for deeper layers of the solar atmosphere (in the chromosphere) are plotted in Fig. 5f. Our calculations show that the spectrum shape is almost independent of the simulation model. This means that the effects of either a converging magnetic field or a self-induced electric field become less significant than collisions, which are the principal mechanism responsible for $\mathrm{HXR}$ emission at this level. In the directions $\theta=0^{\circ}$ and $\theta=90^{\circ}$, the contribution of the emission produced by downward electrons dominates. For the direction $\theta=180^{\circ}$, the contributions of the emission produced by either downward or upward (scattered) electrons are almost equal.

The relative contributions of the scattered and returning electrons into the hard X-ray emission become higher for softer beams (that is shown by blue lines in Fig. 5). In the model taking into account the effects of collisions and self-induced electric field (Fig. 5c), the relative contribution of the return current electrons is more significant for the beam with $\gamma=7$ than for the beam with $\gamma=3$. This is particularly clear for the viewing angles $\theta=90^{\circ}$ (where the emission at $20-100 \mathrm{keV}$ is produced mainly by the return current electrons) and $\theta=0^{\circ}$ (where contributions of the upward and downward electrons are nearly the same at $20-50 \mathrm{keV}$ ); at $\theta=180^{\circ}$, the emission from the return current electrons is strongly dominant both in the hard and soft beams, and a spectral flattening at low energies is observed.

The effect of a converging magnetic field on a softer beam with $\gamma=7$ is similar to that of a harder electron beam with $\gamma=3$ (see Fig. 5d), both qualitatively and quantitatively.

The plots of HXR emission caused by electron beam scattering in the model with collisions + return current + magnetic field convergence (Fig. 5e, blue lines) are similar to those calculated for the model with collisions + return current (Fig. 5c). This implies that for softer beam $(\gamma=7)$, the effect of a self-induced electric field far exceeds all other factors. In particular, the converging magnetic field does not remove the spectral flattening entirely (although it reduces the energy range, where the flattening takes place). The HXR polarization will also be significantly affected, as discussed in Sect. 3.2.2.

In the deeper layers of the solar atmosphere (see Fig. 5f), the emission parameters again become almost insensitive to the simulation model used, because the parameters of the accelerated particles are determined mainly by the collisions here. However, it should be noted that for the beam with $\gamma=7$, only a small fraction of particles can reach the chromosphere. The electrons at greater precipitation depths produce far lower HXR emission intensities (compare the magnitudes of intensity in Figs. 5a and 5f).

\subsubsection{Depth variations of the HXR bremsstrahlung polarization}

With respect to the depth variation in the electron distributions discussed in Sect. 3.1, the polarization degree viewed in a perpendicular direction (right panels at Fig. 5) infers also a strong dependence on precipitation depth and the beam parameters.

At the injection point (Fig. 5a), the polarization degree steadily decreases (by absolute value) with energy for both hard and soft beams. At intermediate precipitation depth, for a harder beam ( $\gamma=3$, shown by red lines) in the model with pure 
collisions (Fig. 5b), the polarization degree has a maximum near the lowest photon energy (at about $15 \mathrm{keV}$ ) and decreases as the energy increases.

For the same beam parameters $(\gamma=3)$ and at the same depth, the model with a magnetic field convergence (Fig. 5d) exhibits an increase in the polarization degree at higher energies (the polarization degree decreases with energy more slowly than without the converging magnetic field) because of the reflected electrons. In contrast, the return current (Fig. 5c) decreases the polarization degree at lower energies, such that the hard electron beam produces emission with nearly constant $(\eta \simeq-0.4)$ polarization in the energy range $12-100 \mathrm{keV}$. A combination of these two factors (see Fig. 5e) results in a weak polarization maximum, which occurs at an energy of about $30 \mathrm{keV}$. In deeper layers (Fig. 5f), the polarization degree decreases in all the models considered, since the electron distribution becomes more isotropic, in general, owing to collisions and pitch-angle diffusion.

The polarization of the emission from the beam with $\gamma=$ 7 (shown by blue lines) is higher than those from beams with $\gamma=3$. This is partially caused by the different numbers of highenergy particles and the properties of the bremsstrahlung crosssections. For the initially injected beam (Fig. 5a), the difference in polarization is caused only by the two aforementioned factors. Another reason is the increase in the degree of scattering of beam electrons for $\gamma=7$, e.g., a larger number of lower energy electrons moves not downwards but in a perpendicular direction. This, in turn, happens owing to the joint effect of collisions and Ohmic losses (Zharkova \& Gordovskyy 2005, 2006).

The converging magnetic field (see Fig. 5d) affects the emission from beams with $\gamma=3$ and $\gamma=7$ in the same way. The effect of a return current (Fig. 5c) for the soft beams is far more significant than for the harder ones: the polarization degree is reduced significantly at lower energies, so we can see the polarization maximum at about $150 \mathrm{keV}$. The combined effects of collisions, converging magnetic field, and return current (Fig. 5e) produce a clearly defined polarization maximum at $50-60 \mathrm{keV}$; the polarization degree at that point exceeds (in absolute value) $60 \%$. The similar polarization maximum, but shifted to lower energies (about $50 \mathrm{keV}$ ) and with lower magnitude, can be seen even in deeper layers (Fig. 5f).

For other viewing angles, the depth variations in HXR polarization produced by a hard beam are, in general, rather complex (see Fig. 6). It can be noted that the precipitation depth of the emitting electrons is the key factor in defining the magnitude of polarization produced. The highest polarization degree $(-46 \%$ at $30 \mathrm{keV}$ and $-37 \%$ at $100 \mathrm{keV}$, for a beam with $\gamma=3$ ) for the initially injected collimated beam is observed in the transversal $\left(\theta=90^{\circ}\right)$ propagation direction. As the electron beam propagates downwards, the polarization dependence on the propagation direction becomes asymmetric, although, in general, the polarization maximum does not deviate much from $\theta \simeq 90^{\circ}$. The polarization values depend on the simulation model. For the model with collisions and return current (Fig. 6a), at $30 \mathrm{keV}$, the polarization degree steadily decreases with depth, which shifts the maximum to lower viewing angles. At $100 \mathrm{keV}$, the polarization degree firstly increases slightly, and then decreases again.

The converging magnetic field increases the rate of electron isotropization leading, thus, to a HXR polarization decrease with depth (see Figs. $6 \mathrm{~b}$ and $6 \mathrm{c}$ ) that occurs far more rapidly than in the homogeneous magnetic field. In the deepest layers, we can see small positive polarization values at the propagation angles $>90^{\circ}$, which are caused by reflected particles with large pitch-angles.
Softer electron beams (Figs. 6d-6f) exhibit a similar dependence of the HXR polarization on the depth and the simulation model. The polarization degree is higher now (up to $-62 \%$ at $30 \mathrm{keV}$ and $-58 \%$ at $100 \mathrm{keV}$ ). As for harder beams, evolution in the polarization degree with depth is governed mainly by the convergence of the magnetic field, but the effect of the return current is now stronger (compare Figs. 6e and 6f).

It can be seen that the HXR polarization calculated even for all energy losses above can still reach $60 \%$ at the right viewing angle $\left(90^{\circ}\right)$ (see Figs. 5-6). This helps one to understand that the position of a flaring atmosphere on the solar surface and the viewing angle from which it is observed can be factors defining the total observed outcome. For example, this can explain the very high polarization degrees (up to $50 \%$ ) reported for the flare 23 July 2002 (McConnell et al. 2003), which was located very close to the limb, and thus seen from the viewing angle close to $90^{\circ}$ and at small integration depth because seen from a side.

\subsubsection{Depth variation of the HXR bremsstrahlung directivity}

The variations with depth of X-ray emission directivity can be explored in detail from Fig. 6. Firstly, we consider the case of $\gamma=3$ (Figs. 6a-6c) when the directivity is the highest. If one includes only the effects of collisions and self-induced electric field (Fig. 6a), then the changes in the X-ray directivity reflect the evolution of electron isotropization via collisions and return current. At the injection point $(\xi=0)$, the emission is highly directed and is radiated mainly downwards. As long as the stream of returning electrons develops at deeper column depths $\left(\xi=10^{18}\right.$ and $\left.10^{19} \mathrm{~cm}^{-2}\right)$, the intensity of emission into the upward hemisphere (towards an observer) increases due to the increased number of particles moving upwards (see Sect. 3.1).

Thus, at lower energies $(30-40 \mathrm{keV})$, the emission becomes almost isotropic. In the deeper layers of the solar atmosphere $\left(\xi=10^{20}\right.$ and $10^{21} \mathrm{~cm}^{-2}$ ), the HXR photon flux decreases because of the lower total number of electrons losing their energy in collisions. This results, in turn, in the density and the energies of the returning electrons decreasing considerably, and the X-ray emission (at $30 \mathrm{keV}$ ) becoming again directed mainly downwards. At higher energies ( $>100 \mathrm{keV})$, this decrease is much less noticeable because only particles with energies much higher than $100 \mathrm{keV}$ can reach this energy at a given depth. These higher energy electrons do not lose their energy fast enough because the Ohmic losses become negligible owing to the lower number of electrons, while collisional losses are reduced because the ambient plasma becoming partially ionized, so the particles can precipitate to deeper atmospheric levels.

Figure $6 \mathrm{~b}$ shows the hard X-ray directivity and polarization for the models with collisions and converging magnetic field. One can see that at lower energy $(30 \mathrm{keV})$, the directivity pattern steadily becomes more isotropic when the electron beam propagates to deeper atmospheric layers. At higher energy $(100 \mathrm{keV})$, the process of isotropization is slower. At the level $\xi=10^{18} \mathrm{~cm}^{-2}$, we can see the emission of two beams, the direct and the mirrored ones.

In Fig. 6c, both the collisions, return current, and magnetic field convergence are taken into account. In this case, the relative contribution of the upward particles into emission increases compared with two previous cases. Now the upward emission can even dominate at $\xi=10^{18}$ and $10^{19} \mathrm{~cm}^{-2}$ for the energy $30 \mathrm{keV}$, and at $\xi=10^{18} \mathrm{~cm}^{-2}$ for the energy $100 \mathrm{keV}$.

It was noted in Sects. 3.1, 3.2, and 3.2.2 that the effects of the self-induced electric field become much stronger for softer electron beams (with $\gamma=7$ ). This conclusion is also confirmed 
V. V. Zharkova et al.: Diagnostics of energetic electrons from HXR. I.
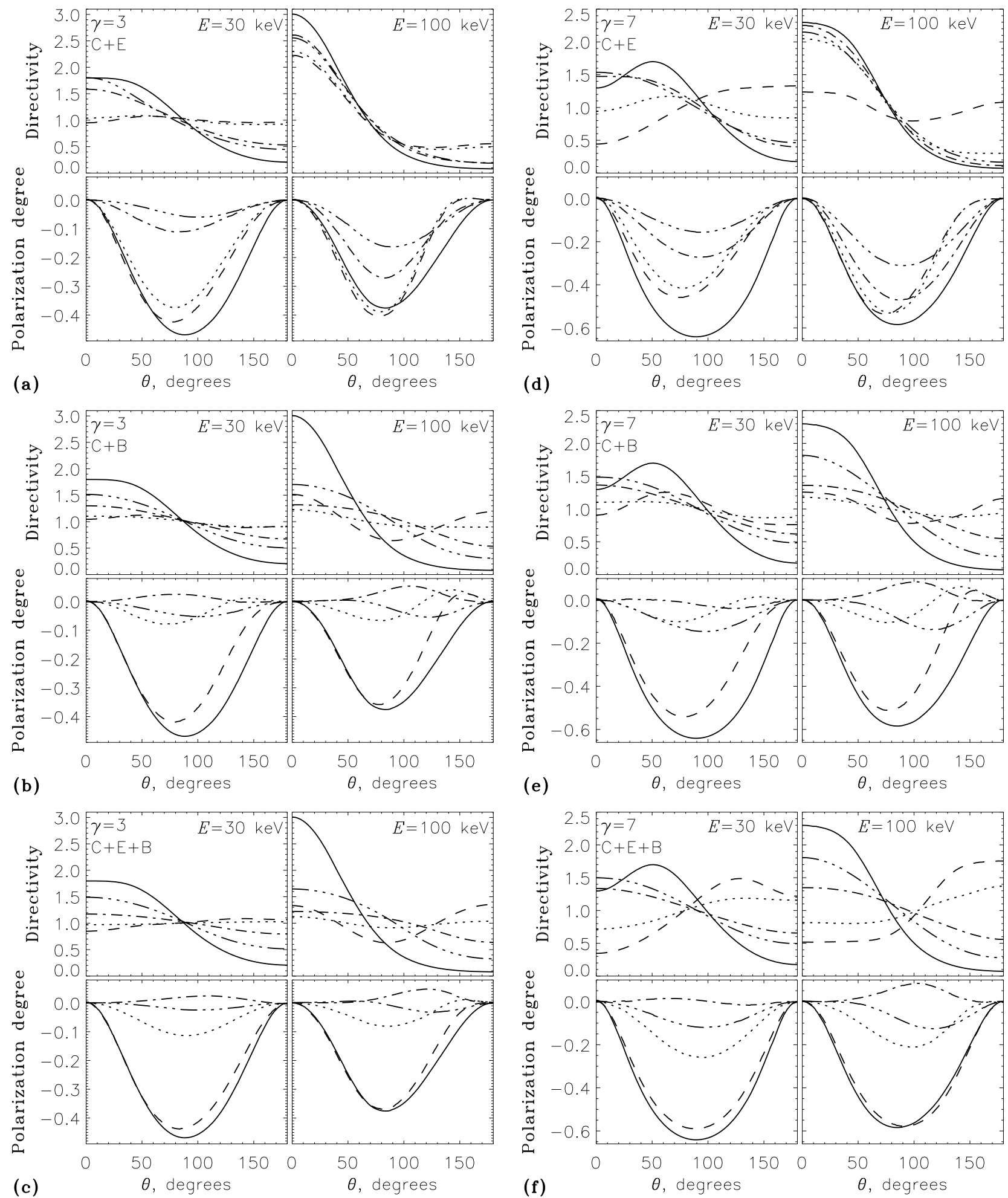

Fig. 6. Directivity and polarization of the hard X-ray emission for different propagation directions. The electron distribution functions are obtained with taking into account different factors (see Fig. 1), the initial power-law indices of beam electrons are $\gamma=3$ (at the left panels) and $\gamma=7$ (at the right panels). Solid line corresponds to the level where $\xi=0$, dashed line $-\xi=10^{18} \mathrm{~cm}^{-2}$, dotted line $-\xi=10^{19} \mathrm{~cm}^{-2}$, dash-dotted line $\xi=10^{20} \mathrm{~cm}^{-2}$, and dash-double-dotted line $-\xi=10^{21} \mathrm{~cm}^{-2}$.

by the directivity plots (Figs. 6d-6f). In Fig. 6d (collisions + return current), one can see that the X-rays are emitted mainly upwards at $\xi=10^{18} \mathrm{~cm}^{-2}$ and $30 \mathrm{keV}$; the upward emission at the same depth at $100 \mathrm{keV}$ is comparable to the downward one. The converging magnetic field (Fig. 6e) has almost the same effect as for the harder beam. In Fig. 6f (collisions + return current + magnetic field convergence), the upward emission strongly dominates at the levels of $\xi=10^{18}$ and $10^{19} \mathrm{~cm}^{-2}$ for both considered energies. These effects need to be incorporated into the integrated polarization to be compared with those measured by the RHESSI instruments. 

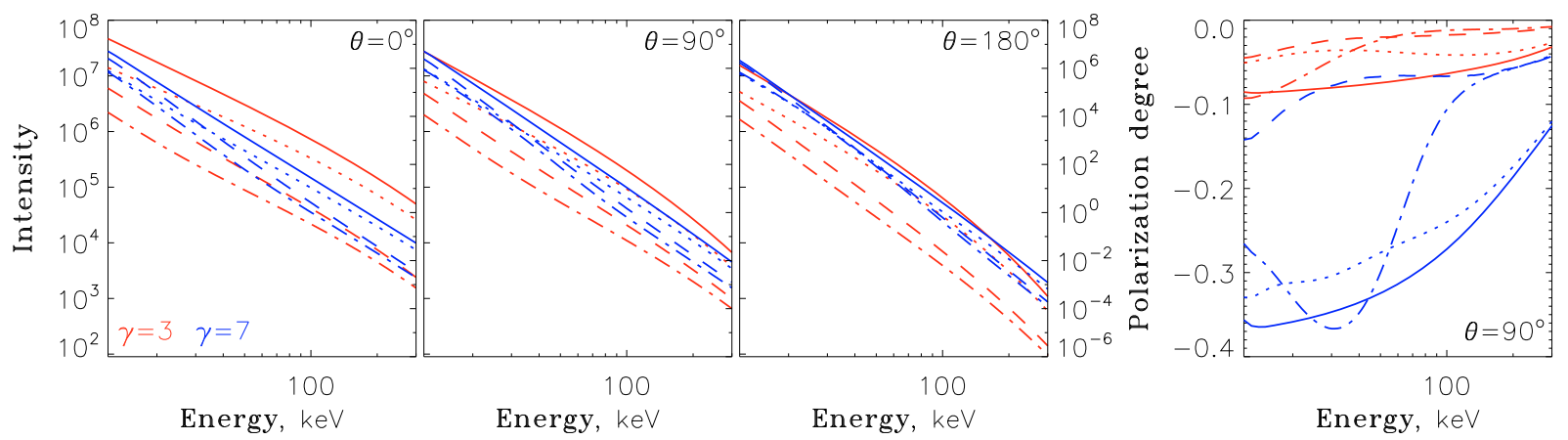

Fig. 7. Integrated by column depth intensity (in relative units) and polarization of hard X-ray emission for different energies. The initial power-law indices of the beam electrons are: $\gamma=3$ (red lines) and $\gamma=7$ (blue lines). The intensity profiles for $\gamma=3$ and $\gamma=7$ are indicated by using different scaling: the intensity magnitudes are shown on the left (for $\gamma=3$ ) and right (for $\gamma=7$ ) margins, respectively. Different lines correspond to the different simulation models: solid line - the case of pure collisions (C), dotted line - collisions and return current are taken into account $(\mathrm{C}+\mathrm{E})$, dashed line - collisions and converging magnetic field are taken into account $(\mathrm{C}+\mathrm{B})$, and dash-dotted line - all factors taken into account $(\mathrm{C}+\mathrm{E}+\mathrm{B})$.

\subsubsection{Integrated HXR bremsstrahlung emission and polarization}

The total emission from the considered coronal magnetic tube with the emission parameters integrated over all layers using Eq. (7), is shown in Figs. 7 and 8. As in Fig. 5, the emission parameters for the electron beams with different power-law indices in Fig. 7 are shown in different colors (red for $\gamma=3$ and blue for 7). We note that in Fig. 7, the intensity plots for $\gamma=3$ and $\gamma=7$ are drawn for different scalings (shown at the left axis for $\gamma=3$ and at the right axis for $\gamma=7$ ).

One can note from Fig. 7 that a harder beam exhibits a strong dependence on magnetic field convergence (compare the models with a constant magnetic field $(\mathrm{C}, \mathrm{C}+\mathrm{E})$ with those taking into account a magnetic field convergence $(\mathrm{C}+\mathrm{B}, \mathrm{C}+\mathrm{E}+\mathrm{B}))$. In the converging magnetic field, the emission intensity is lower than in the homogeneous magnetic field, because the magnetic mirroring reduces the number of electrons reaching the deepest layers of the solar atmosphere. For the softer beams, this dependence is less significant, as the number of electrons reaching the deepest layers is small in any case due to collisional damping. A small spectral flattening at lower energies is evident, although it is less pronounced than for the emission from the individual layers in the corona (compare with Fig. 5); the "break" energy (for the $\mathrm{C}+\mathrm{E}+\mathrm{B}$ model and for the upward emission) is found to be about $100 \mathrm{keV}$ for the beam with $\gamma=3$ and about $50 \mathrm{keV}$ for $\gamma=7$. This confirms the analytical predictions for a simplified precipitation model done by Zharkova \& Gordovskyy (2006).

The integrated HXR directivity and polarization are found to be even more sensitive to the beam parameters and viewing angles. One can see that for harder beams, the return current almost does not change the integrated directivity (in comparison with the purely collisional model), but reduces the integrated polarization at both energies considered. The converging magnetic field reduces the directivity and polarization significantly, and the results for the $\mathrm{C}+\mathrm{E}+\mathrm{B}$ model are similar to those for $\mathrm{C}+\mathrm{B}$.

For softer beams, the effect of the return current is much stronger. We can see that the combination of the converging magnetic field and return current changes the directivity at $30 \mathrm{keV}$, so the integrated emission is directed mainly upwards. The integrated polarization in this case can become higher than for the purely collisional model. At $100 \mathrm{keV}$, the downward emission always dominates, but the upward emission in $\mathrm{C}+\mathrm{E}+\mathrm{B}$ model is considerably stronger than in the other three models. It can be seen from Figs. 7, 8 that the maximal polarization

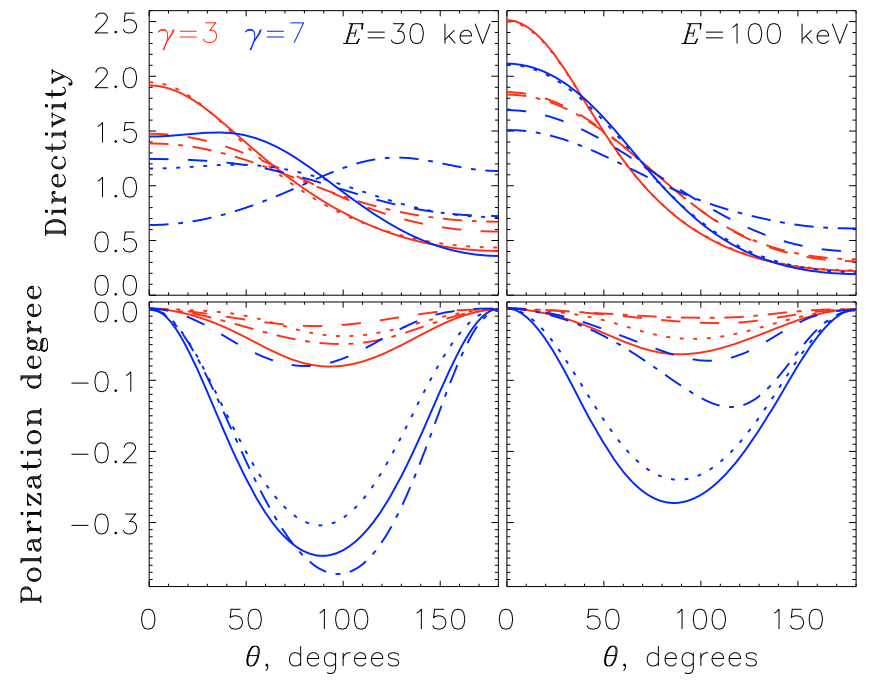

Fig. 8. Directivity and polarization of the hard X-ray emission for the different propagation directions. The emission parameters are integrated over all layers of coronal magnetic tube. Initial power-law indices of the accelerated particles are: $\gamma=3$ (red lines) and $\gamma=7$ (blue lines). Different lines correspond to the different simulation models (see Fig. 7).

degree (about $-36 \%$ ) is achieved for the beam with $\gamma=7$, in the model with collisions, return current, and converging magnetic field, at the energy of about $30 \mathrm{keV}$ and transversal propagation direction.

For a hard electron beam $(\gamma=3)$ of medium intensity $\left(F=10^{10} \mathrm{erg} \mathrm{cm}^{-2} \mathrm{~s}^{-1}\right)$, the parameters of HXR emission are defined by the electrons, which do not induce a strong electric field and lose their energy mainly by collisions, i.e., in other words, for harder beams their X-ray emission is generated mainly in the deepest layers of the solar atmosphere (transition region and chromosphere). In contrast, for the electron beams with $\gamma=7$, only a small fraction of particles can reach the chromosphere. As a result, the X-ray emission from these beams is generated mainly in the corona, where, in turn, the effect of the selfinduced electric field is significant.

The current study has also established that the measurements of HXR integrated polarization combined with those of photon flux can derive far more precise electron beam parameters and provide far deeper insight into the mechanisms of particle 
V. V. Zharkova et al.: Diagnostics of energetic electrons from HXR. I.
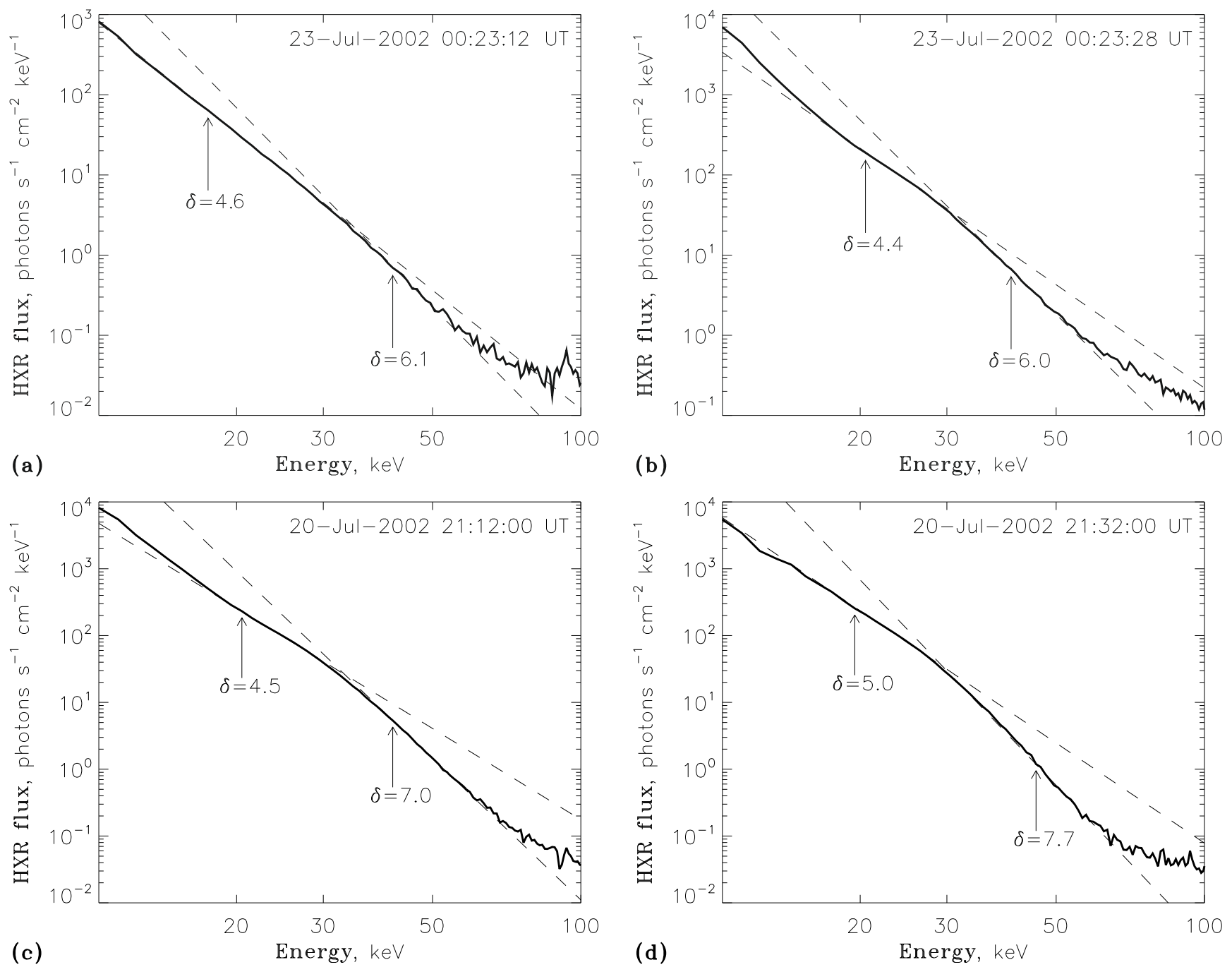

Fig. 9. The hard X-ray double power-law spectra observed by RHESSI for the flares of 23 July 2002 (upper plots) and 20 July 2002 (lower plots) for the times plotted in the top right corners of each plot. The top left and right plots correspond to the electron energy fluxes of $2 \times 10^{10}$ and $5 \times 10^{10} \mathrm{erg} \mathrm{cm}^{-2} \mathrm{~s}^{-1}$ for the electron spectral index $\gamma=7$, respectively. The bottom left and right plots correspond to the energy fluxes of $1 \times 10^{11}$ and $5 \times 10^{10} \mathrm{erg} \mathrm{cm}^{-2} \mathrm{~s}^{-1}$, respectively. The straight lines show the fits by single power law spectra for lower energy and upper energy parts.

transport in the deeper atmospheric levels than the pure emission measurements.

\subsubsection{Comparison with observations}

We now compare our simulation results with the available observations of HXR bremsstrahlung emission, polarization, and directivity.

\section{HXR bremsstrahlung photon spectra}

We first consider the deduced photon energy spectra and determine how they can be reproduced by the theoretical calculations (Fig. 9). It can be seen that the observed energy spectra for the flares 20 and 23 July 2002 have distinctive double power-law energy spectra where the lower energy part is flattened to much lower spectral indices (e.g., 4.6 and 4.4 for the 23 July flare (Sui et al. 2007) and 4.5 and 5.0 for the 20 July flare) compared to the higher energy parts (6.1-6.2 for the 23 July flare (Holman et al. 2003) and 7-7.7 for the 20 July flare). The observed HXR photon spectra can be well reproduced by the simulated ones produced by the distribution functions of precipitating and returning electrons by using Eq. (8) for the relativistic cross-sections from Appendix A and taking into account the self-induced electric field that causes the photon spectrum flattening at lower energies (Zharkova \& Gordovskyy 2006) as discussed below.

The theoretical spectra of HXR bremsstrahlung photon spectra are shown to be divided into two parts, lower and higher energy ones with much lower spectral indices at the lower energy parts than in the higher ones (see Fig. 11 in Zharkova \& Gordovskyy 2006). The break energy corresponds to the electric field induced by lower energy electrons losing their energy in collisions and Ohmic losses. It can be seen that the difference in the spectral indices $\delta_{1}$ of photon spectra at the lower energy part is greater for beam electrons with higher initial spectral index $\gamma$ and initial energy flux $F_{0}$. In contrast, the photon spectral indices in the higher energy part of the spectrum $\delta_{\mathrm{u}}$ are lower than the initial spectral indices of beam electrons $\gamma$ for lower initial energy fluxes $F_{0}$ of beam electrons, or equal to $\gamma$ for beams with a higher initial energy flux of $10^{12} \mathrm{erg} \mathrm{cm}^{-2} \mathrm{~s}^{-1}$.

This means that for the flare on 23 July 2002, where the lower energy index changes from 4.6 to 4.4 in the left and right top plots of Fig. 9, respectively (Holman et al. 2003), one observes an increase in the initial energy flux of beam electrons in the two consecutive times by a factor $2-$ from $2 \times 10^{10}$ to $4 \times 10^{10} \mathrm{erg} \mathrm{cm}^{-2} \mathrm{~s}^{-1}$, which is confirmed by the light curves 

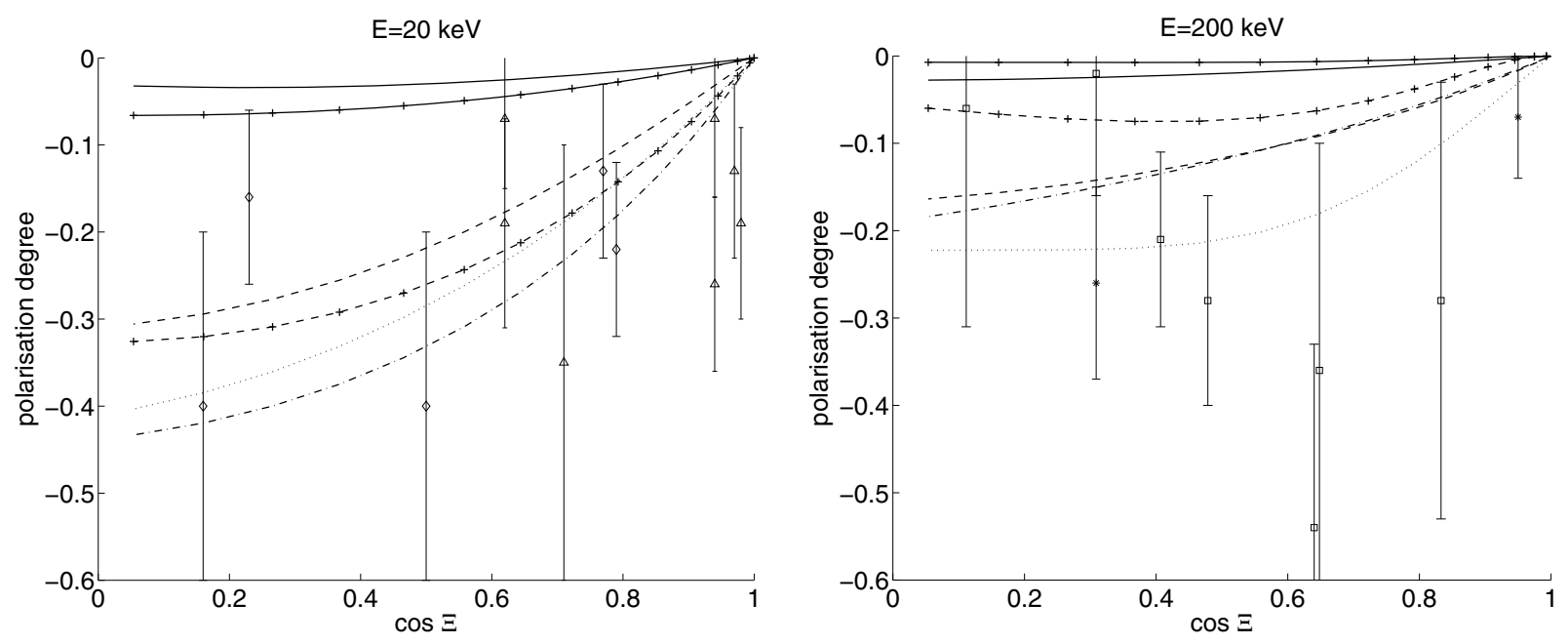

Fig. 10. Comparison of the simulations of HXR bremsstrahlung polarization for $20 \mathrm{keV}$ (left plot) and $200 \mathrm{keV}$ (right plot) produced for different position angle on the solar disk ( $\cos \Xi=1$ in the disk center and 0 on the limb) by a wider electron beam with $\Delta \mu=0.2$ and the energy flux of $10^{10} \mathrm{erg} \mathrm{cm}^{-2} \mathrm{~s}^{-1}(\gamma=3: \mathrm{C}+\mathrm{E}$ model - solid line, $\mathrm{C}+\mathrm{E}+\mathrm{B}$ model - solid line with crosses; $\gamma=7$ : $\mathrm{C}+\mathrm{E}$ model - dashed line, $\mathrm{C}+\mathrm{E}+\mathrm{B}$ model - dashed line with crosses) and by more collimated $(\Delta \mu=0.02)$ electron beam $(\mathrm{C}+\mathrm{E}$ model $)$ with $\gamma=7$ and the initial energy fluxes of $10^{10}$ (dot-dashed lines) and $10^{12} \mathrm{erg} \mathrm{cm}^{-2} \mathrm{~s}^{-1}$ (dotted lines). The observation results are plotted as follows: diamonds correspond to the observations of Tindo et al. (1970, 1972a,b) at $15 \mathrm{keV}$, triangles - to the observations of Tramiel et al. (1984) at 16-21 keV, squares - to the observations of Suarez-Garcia et al. (2006) at 100-350 keV, and asterisks - to the observations of Boggs et al. (2006) at 200-400 keV.

for this flare (Holman et al. 2003). This increase in the electron energy flux for the two observed cases results in a stronger flattening of the photon spectrum in the second case than in the first.

A spectral difference between the lower and upper spectral indices $\delta_{1}$ and $\delta_{\mathrm{u}}$ of the observed photon spectra for these instances (fits to the simulated spectra plotted by dashed lines in Fig. 9) increased from $1.5(6.1-4.6)$ to $1.6(6.0-4.4)$. For the 20 July 2002 flare, the spectral index difference in the photon spectra produced by beam electrons plotted in the bottom graphs of Fig. 9 increases from 4.5 to 5.0. This confirms that the energy flux of beam electrons increased in time from $1 \times 10^{11}$ to $5 \times 10^{11} \mathrm{erg} \mathrm{cm}^{-2} \mathrm{~s}^{-1}$ as indicated in Fig. 11 and Table 2 in the paper by Zharkova \& Gordovskyy (2006). Thus, we reckon that the observed spectral flattening at lower energies and the softhard-soft pattern in the lower energy spectral indices of photon spectra can be naturally explained by the low-high-low variations in the initial energy flux of beam electrons.

We note that the above-mentioned spectral flattening of the $\mathrm{X}$-ray spectra at lower energies caused by the returning beam electrons is qualitatively similar to the expected influence of the so-called albedo effect (e.g., Bai \& Ramaty 1978; Kontar et al. 2006). Part of the downwardly emitted photons should be reflected from the photosphere up to the observer, thus distorting the primary emission spectra. The albedo contribution should be highest for photon energies of about $30-50 \mathrm{keV}$ (owing to the energy dependence of the Compton scattering probability) and for events occurring close to the solar disk center (due to the geometrical reasons). The same can be said about the contribution of the returning electrons. In both cases we consider the reflection process, but either the photons or the precipitating electrons (and photons emitted by them) can change their propagation direction.

\section{HXR bremsstrahlung directivity and polarization}

Other properties of the HXR bremsstrahlung emission that can help us to understand more clearly the mechanisms of electron precipitation into a flaring atmosphere is linear polarization calculated from Eq. (8) and directivity obtained from Eq. (9). To achieve a closer fit to observations, we considered the electron beam with all energy losses (collisions, convergence, and Ohmic losses) and with wider and narrower pitch angle dispersion $\Delta \mu=0.2,0.02$ in the initial distribution defined by Eq. (2). The polarization calculated for HXR bremsstrahlung photon energies of $20 \mathrm{keV}$ and $200 \mathrm{keV}$ for different models of electron beam precipitation and flare locations on the solar disk is plotted in Fig. 10 and the directivity in Fig. 11. The simulations are tested by numerous observations of HXR polarization and directivity plotted in each figure with their error bars as discussed below.

We note that the polarization is higher by up to a factor 2 for lower energy photons ( $20 \mathrm{keV}$ ) than for higher energy ones (200 keV), which may be caused by the smaller number of high energy photons compared to the lower energy ones because of the power-law dependence on energy. For both photon energies (20 and $200 \mathrm{keV}$ ), the simulated HXR polarization smoothly increases towards the solar limb. For beam electrons with a wider pitch-angle dispersion $\Delta \mu=0.2$, this increase is greater for a higher spectral index of 7 compared to 3 and for the models $\mathrm{C}+\mathrm{E}+\mathrm{B}$ taking into account magnetic field convergence in addition to the model with collisions plus electric field $(\mathrm{C}+\mathrm{E})$. The same is valid for the polarization in high energy band of $200 \mathrm{keV}$ produced by beams with higher energy flux of $10^{12} \mathrm{erg} \mathrm{cm}^{-2} \mathrm{~s}^{-1}$ in the right plot of Fig. 10. For the polarization at lower energy of $20 \mathrm{keV}$, the higher the initial energy flux of beam electrons, the lower the polarization (see left plot in Fig. 10). The latter is a clear electric field effect that causes more beam electrons to return back to the corona and a greater spectral flattening for $\gamma=7$ as discussed above. For a narrower electron beam, HXR polarization increases closer to the limb by $30-40 \%$.

To compare HXR polarization with available, observations we considered our theoretical curves to reproduce the observed polarization well if the observational error bars appeared within the simulated polarization curves. The comparison is complicated by availability only of polarization degrees and the absence of photon spectra and spectral indices in the polarization reports by some authors. Thus, we have not yet had the chance to compare all our simulations directly with observations for a 

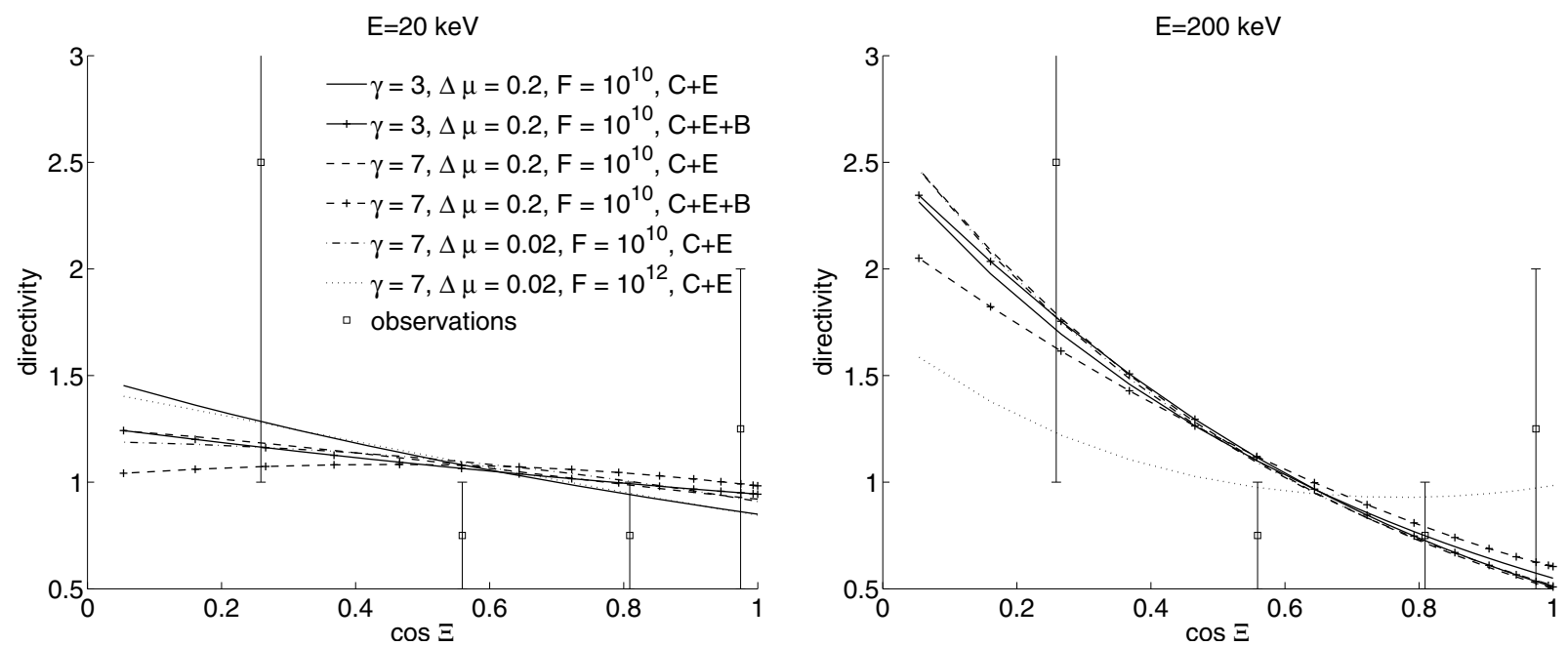

Fig. 11. A comparison of the directivity estimations inferred for energies $20-200 \mathrm{keV}$ from the statistical survey of different flare positions at the solar disk ( $\cos \Xi=1$ in the disk center and 0 on the limb) at $\sim 20-100 \mathrm{keV}$ by Kašparová et al. (2007) with the calculated directivity $\left(I_{\odot} / I_{\oplus}\right)$ of hard X-ray bremsstrahlung emission produced by the model electron beams with the same parameters as in Fig. 10.

single flare, but only a collection for many flares. This is why we present the distribution of polarization degree that depends on the position of a flare on the solar disk for a set of electron beam indices and energy fluxes, and plot the observed polarization on the same graph. It is clearly seen that in most cases the measured polarization degrees are located well between the theoretical curves, confirming the validity of the applied models of electron precipitation.

Although, there are some discrepancies between the simulations and observations located at the disk center, which can be caused by the loop tilts which lead to the resulting viewing angle being higher than their position angle on the disk. Nevertheless, at lower $(20 \mathrm{keV})$ and higher energy $(>200 \mathrm{keV})$, the fit is significantly improved (see the right plots in Fig. 10) by consideration of a narrower electron beam, which leaves only one measurement at $\cos \Xi \simeq 0.55$ not covered by the simulated curves. The only measurement that is not fitted by the simulations is that of the heliocentic angle of about 50 degrees, which can be produced by even more focused beams, say with $\Delta \mu=0.008-0.01$, or by the albedo effect (which, according to Bai \& Ramaty 1978, can also increase the visible polarization).

The fit of our theoretical predictions of the HXR bremsstrahlung directivity to those measured by Kašparová et al. (2007) is plotted in Fig. 11. The simulations are carried out for both wide and narrow electron beams with $\Delta \mu=0.2$ and 0.02 for the same beam parameters discussed for polarization (Fig. 10). It can be noted that the directivity of lower energy photons of $20 \mathrm{keV}$ is lower and less widely dispersed over locations across the solar disk than those of higher energy, e.g. $200 \mathrm{keV}$. The directivity is at its highest approaching 2.5 at the solar limb, while it smoothly decreases to 0.5 at the solar disk center. The directivity of harder beams $(\gamma=3)$ for models with either pure collisions or collisions and electric field, is higher than for softer beams $(\gamma=7)$, and for models considering also a magnetic field convergence. The directivity of higher energy photons produced by a narrower beam with a higher initial energy flux becomes much lower than that for a wider beam with lower energy flux.

The fit to the observed directivity to the theoretical curve of directivity produced for the models $\mathrm{C}+\mathrm{E}$ by a narrower electron beam with higher energy flux of $10^{12} \mathrm{erg} \mathrm{cm}^{-2} \mathrm{~s}^{-1}$, is satisfactory for higher energy emission (the right plot in Fig. 11). We emphasize that in the current paper we have not taken into account any albedo effects, and considered only the non-isotropic pitch angle scattering of beam electrons during their precipitation in a converging magnetic field and self-induced electric field. The effect of particle kinetics is exhibited in their emission properties and, in particular, its polarization and directivity.

However, when interpreting solar X-ray observations, the albedo and electric field effects can substantially change the HXR directivity and polarization since they act on HXR emission in similar ways. This explains why Kašparová et al. (2007) concluded that the observed deviations of the RHESSI flare $\mathrm{X}$-ray spectra from the power-law ones can be well explained by the albedo effect, as for our current and previous conclusions about electric field effect (Zharkova \& Gordovskyy 2006). Sui et al. (2007) found that the albedo correction is usually insufficient to explain the observations, and the remaining difference is most probably due to the return current. A clearer differentiation between the albedo and return current effects can be achieved, on the one hand, by using the spatially resolved observations of very compact X-ray sources (the albedo source should be larger than the primary X-ray source). However, simultaneous simulations of the albedo and return current contributions are urgently required and will be presented in a forthcoming paper.

\section{Conclusions}

The distribution functions of power-law beam electrons within the energy range of $12 \mathrm{keV}$ to $1.2 \mathrm{MeV}$ are simulated during their precipitation into a flaring atmosphere by using the FokkerPlanck approach and taking into account collisional and Ohmic energy losses in a converging magnetic field. By using the obtained electron distributions, the resulting HXR emission with relativistic bremsstrahlung cross-sections, directivity and polarization are also calculated for precipitating and returning particles with the positive and negative pitch angle cosines, respectively, at various depths and for different viewing angles. The conclusions of their comparison are summarized below.

The distributions of beam electrons are shown to be strongly affected by their self-induced electric field and magnetic field convergence at given depths leading to a formation of particle streams moving upwards to the source where they were injected from. The self-induced electric field produces the returning electrons, or return current, which compensates the electric field 
induced by precipitating electrons. For the extended upper cutoff energy of $1.2 \mathrm{MeV}$, the energy of returning electrons cover the range up to $300 \mathrm{keV}$ and their distributions are quasi-thermallike with wide maxima being caused by pitch-angle diffusion at $80 \mathrm{keV}$ for $\gamma=3$ and $120 \mathrm{keV}$ for $\gamma=7$. For a softer beam, the number of returning electrons at some depths can exceed the number of precipitating ones.

A converging magnetic field with a reasonable convergence coefficient (a factor of 2-3) towards the characteristic depth in the upper chromosphere $\left(\xi=10^{20} \mathrm{~cm}^{-2}\right)$ produces a stream of mirrored electrons that are reflected by the magnetic field within the loss cone defined by this convergence. The effect of a converging magnetic field becomes noticeable only after the initial well-collimated beam electrons (with the pitch-angle dispersion $\Delta \mu=0.2$ centered about $\mu=1$ ) become scattered by collisions to larger pitch angles, or smaller $\mu$ that only happens after quite a few steps in the precipitation depth and lasts until the convergence exists. The energy spectra of electrons reflected by a converging magnetic field remain the same (power-law like) as for the precipitating electrons. The convergence effect is found to be more important for a harder beam than for a softer one which is more affected by a self-induced electric field. The joint effect of self-induced electric and converging magnetic fields at all precipitation depths creates more electrons that return back to the corona, whose numbers are higher for softer beams.

The beams, both precipitating and returning, produce HXR bremsstrahlung emission throughout their precipitation depths. It was found that at higher atmospheric levels $\left(\xi \leq 2 \times 10^{18} \mathrm{~cm}^{-2}\right)$ the emission from precipitating electrons is often comparable or even lower than those from the returning electrons. This is caused by the self-induced electric field (for softer beams) or by the magnetic field convergence (for harder beams).

The effect of a converging magnetic field is strongest for a viewing angle of $180^{\circ}$ and decreases for smaller viewing angles. Similarly, the effect of a self-induced electric field is strongest for the viewing angles about $180^{\circ}$, but is significant for other directions as well. The joint effects of all energy losses on HXR emission are defined by the dominant mechanisms of energy losses, e.g., Ohmic losses prevailing for softer beams and converging magnetic field prevailing for harder beams. The HXR emission observed in the upper corona at small viewing angles (less then $30^{\circ}$ ) is produced predominantly by returning electrons, not by the precipitating ones. For lower atmospheric depths, the effects of external fields are reduced and the emission is defined by collisions only.

The depth and viewing angle variations of HXR polarization are far more pronounced than those of the emission. Near the electron injection site, the polarization is maximal at the lower energy cutoff and then shifts to higher energies with every precipitation depth, approaching $25 \mathrm{keV}$ for models with pure collisions and $100 \mathrm{keV}$ for models with collisions and a return current. In deeper layers, the polarization decreases because of the isotropization of electrons by collisions. The maximum polarization is observed at the viewing angle of $90^{\circ}$ and shifts to lower angles for softer beams. The polarization of HXR emission caused by soft electron beams at $90^{\circ}$ at higher depths is higher than those produced by harder beams because the former have more electrons scattered to larger pitch angles at smaller depths. In deeper atmospheric levels, the polarization for harder beams becomes higher that for softer ones, as defined by the electron isotropization by collisions, which is higher for softer electrons.

The HXR directivity, which determines the relative importance of downward and upward emission, is also shown to be a function of precipitating depth and viewing angle being controlled by a combination of the flare position on the solar disk and the tilt of the flaring loop towards the solar surface. At the injection point $(\xi=0)$, the emission is highly directed and radiated mainly downwards. For all simulation models, in the deeper atmospheric layers (about $\xi=10^{18} \mathrm{~cm}^{-2}$ ), a stream develops from the electrons returning upwards to the source resulting in an increase in the HXR intensity and directivity for small viewing angles in the upward direction (towards an observer).

For the models with an electric field and collisions, at upper atmospheric levels, the HXR emission at lower energies $(30 \mathrm{keV})$ is directed more upwards than downwards, owing to the increased number of particles moving upwards. In the deeper layers of the solar atmosphere $\left(\xi=10^{20}\right.$ and $\left.10^{21} \mathrm{~cm}^{-2}\right)$, the upward HXR photon flux (at $30 \mathrm{keV}$ ) is lower because of a considerably lower number of returning electrons leading to the X-ray emission being again directed mainly downwards. At higher energies $(100 \mathrm{keV})$, this emission is directed downwards because this effect is less pronounced and the particles do not lose their energy fast enough in the partially ionized plasma, so they can precipitate to deeper atmospheric levels.

For the models with collisions and converging magnetic field, the directivity pattern of HXR bremsstrahlung emission remains nearly the same when the electron beam propagates into deeper atmospheric layers showing that the emission is directed mainly downwards. For models including collisions, return current, and magnetic field convergence, the relative contribution into the HXR emission of the particles moving upwards increases compared to the case of collisions and Ohmic losses. In this case, the directivity is also governed by the self-induced electric field and collisions, but at smaller viewing angles (towards the observer) it becomes slightly higher owing to magnetic mirroring. At larger viewing angles, the emission parameters are fully defined by the electric field and collisions, so the directivity is very different from that caused by magnetic convergence. This difference can be used to diagnose these effects in the observations.

The total (spatially integrated) HXR emission spectra and directivity also show a dependence on both the beam parameters and the energy losses. For softer beams, there is a noticeable flattening of the photon spectra at lower energies caused by the selfinduced electric field, which increases for larger viewing angles approaching a maximum at the angle of $180^{\circ}$. There is also some dependence on magnetic field convergence for harder beams that makes the corresponding photon spectra softer at smaller viewing angles, while at larger angles they show no significant dependence on the convergence factor. These dependencies are clearly controlled by the dynamics of energy losses for harder and softer beams, the former being more sensitive to magnetic mirroring and the latter to Ohmic losses.

The proposed precipitation model with collisional and Ohmic losses for an electron beam with wider pitch angle dispersion of 0.2 allowed us to fit the double power law HXR photon spectra with a spectrum flattening at lower energies observed in the flares of 20 and 23 July 2002. The observed spectral flattening at lower energies and the soft-hard-soft pattern in the lower energy spectral indices of photon spectra can be naturally explained by the low-high-low variations in the initial energy flux of injected beam electrons. The observed directivity of HXR photons of $20 \mathrm{keV}$ derived for a large number of flares located between the disk center and limb is also reproduced well by the theoretical directivity calculated for an electron beam with a very narrow pitch angle dispersion of 0.02 . The simulated polarization of this narrow-directed electron beam fits up to $90 \%$ of 
V. V. Zharkova et al.: Diagnostics of energetic electrons from HXR. I.

all the available polarimetric and directivity observations carried out at various locations on the solar disk.

Thus, the current study allows us to establish that polarization measurements combined with the observations of HXR photon flux can help researchers to extract the electron beam parameters far more precisely and to obtain a much deeper insight into the mechanisms of particle transport into deeper atmospheric levels than attainable from only measurements of HXR bremsstrahlung emission (such as light curves and energy spectra).

Acknowledgements. The authors would like also to express their deepest gratitude to the Royal Society (UK) for providing a visiting grant for AK to visit the University of Bradford in March-April 2008 where the paper was initiated. The authors would like to thank the anonymous referee and the Editor for their constructive comments from which paper strongly benefited. The work was partially supported by the Royal Society (UK), the Science, Technology and Facilities Council (UK) (grant PP/E001246/1) and the Russian Foundation of Basic Research (grant 09-02-92610).

\section{Appendix A: Hard X-ray bremsstrahlung cross-section}

The parameters $\sigma_{I}$ and $\sigma_{Q}$ in Eqs. (6), (7) can be expressed using the bremsstrahlung cross-sections (Bai \& Ramaty 1978; Leach \& Petrosian 1983)

$\sigma_{I}(h v, \theta, E, \mu, \varphi)=\frac{\mathrm{d}^{2} \sigma_{\perp}}{\mathrm{d}(h v) \mathrm{d} \Omega}+\frac{\mathrm{d}^{2} \sigma_{\|}}{\mathrm{d}(h v) \mathrm{d} \Omega}$,

$\sigma_{Q}(h v, \theta, E, \mu, \varphi)=\left[\frac{\mathrm{d}^{2} \sigma_{\perp}}{\mathrm{d}(h v) \mathrm{d} \Omega}-\frac{\mathrm{d}^{2} \sigma_{\|}}{\mathrm{d}(h v) \mathrm{d} \Omega}\right] \cos 2 \chi$,

where $\mathrm{d}^{2} \sigma_{\|} /[\mathrm{d}(h v) \mathrm{d} \Omega]$ and $\mathrm{d}^{2} \sigma_{\perp} /[\mathrm{d}(h v) \mathrm{d} \Omega]$ are, respectively, the bremsstrahlung cross-sections for the photons with polarization parallel and perpendicular to the emission plane, that is, with respect to the plane given by the vector of electron impulse and the wave vector of $\mathrm{X}$-rays. The Stokes parameter $Q$ is usually calculated with respect to the normal plane, that is, to the plane given by the magnetic field and the wave vector of X-rays. The parameter $\chi$ is the angle between the normal and the emission planes

$\cos 2 \chi=1-\frac{2 \sin ^{2} \alpha \sin ^{2} \varphi}{\sin ^{2} \psi}$,

where $\psi$ is the angle between the vector of electron impulse and the wave vector of $\mathrm{X}$-rays, this angle being determined by the equation

$\cos \psi=\cos \alpha \cos \theta+\sin \alpha \cos \varphi \sin \theta$.

The expressions for the bremsstrahlung cross-sections in the relativistic case are given in the articles of Gluckstern \& Hull (1953) and Bai \& Ramaty (1978) by

$$
\begin{aligned}
\frac{\mathrm{d}^{2} \sigma_{\|}}{\mathrm{d}(h v) \mathrm{d} \Omega}= & \frac{Z^{2}}{8 \pi} \frac{r_{0}^{2}}{137} \frac{p_{1}}{p_{0}} \frac{Q_{E}}{h v}\left\{\frac{8 \sin ^{2} \psi\left(2 \Gamma_{0}^{2}+1\right)}{p_{0}^{2} \Delta^{4}}\right. \\
& -\frac{\left(5 \Gamma_{0}^{2}+2 \Gamma_{0} \Gamma_{1}+5\right)}{p_{0}^{2} \Delta^{2}}-\frac{\left(p_{0}^{2}-k^{2}\right)}{T^{2} \Delta^{2}}+\frac{2\left(\Gamma_{0}+\Gamma_{1}\right)}{p_{0}^{2} \Delta} \\
& +\frac{L}{p_{0} p_{1}}\left[\frac{4 \Gamma_{0} \sin ^{2} \psi\left(3 k-p_{0}^{2} \Gamma_{1}\right)}{p_{0}^{2} \Delta^{4}}\right. \\
& +\frac{2 \Gamma_{0}^{2}\left(\Gamma_{0}^{2}+\Gamma_{1}^{2}\right)-\left(9 \Gamma_{0}^{2}-4 \Gamma_{0} \Gamma_{1}+\Gamma_{1}^{2}\right)+2}{p_{0}^{2} \Delta^{2}}
\end{aligned}
$$

$$
\begin{aligned}
& \left.+\frac{k\left(\Gamma_{0}^{2}+\Gamma_{0} \Gamma_{1}\right)}{p_{0}^{2} \Delta}\right] \\
& +\frac{\varepsilon^{T}}{p_{1} T}\left[\frac{4}{\Delta^{2}}-\frac{7 k}{\Delta}-\frac{k\left(p_{0}^{2}-k^{2}\right)}{T^{2} \Delta}-4\right]-\frac{4 \varepsilon}{p_{1} \Delta} \\
& +\frac{1}{p_{0}^{2} \sin ^{2} \psi}\left[\frac{2 L}{p_{0} p_{1}}\left(2 \Gamma_{0}^{2}-\Gamma_{0} \Gamma_{1}-1-\frac{k}{\Delta}\right)\right. \\
& \left.\left.-\frac{4 \varepsilon^{T}\left(\Delta-\Gamma_{1}\right)^{2}}{p_{1} T}-\frac{2 \varepsilon\left(\Delta-\Gamma_{1}\right)}{p_{1}}\right]\right\},
\end{aligned}
$$

$$
\begin{aligned}
\frac{\mathrm{d}^{2} \sigma_{\perp}}{\mathrm{d}(h v) \mathrm{d} \Omega}= & \frac{Z^{2}}{8 \pi} \frac{r_{0}^{2}}{137} \frac{p_{1}}{p_{0}} \frac{Q_{E}}{h v}\left\{\frac{-\left(5 \Gamma_{0}^{2}+2 \Gamma_{0} \Gamma_{1}+1\right)}{p_{0}^{2} \Delta^{2}}-\frac{\left(p_{0}^{2}-k^{2}\right)}{T^{2} \Delta^{2}}\right. \\
& -\frac{2 k}{p_{0}^{2} \Delta}+\frac{L}{p_{0} p_{1}}\left[\frac{2 \Gamma_{0}^{2}\left(\Gamma_{0}^{2}+\Gamma_{1}^{2}\right)-\left(5 \Gamma_{0}^{2}-2 \Gamma_{0} \Gamma_{1}+\Gamma_{1}^{2}\right)}{p_{0}^{2} \Delta^{2}}\right. \\
& \left.+\frac{k\left(\Gamma_{0}^{2}+\Gamma_{0} \Gamma_{1}-2\right)}{p_{0}^{2} \Delta}\right]+\frac{\varepsilon^{T}}{p_{1} T}\left[\frac{k}{\Delta}-\frac{k\left(p_{0}^{2}-k^{2}\right)}{T^{2} \Delta}+4\right] \\
& -\frac{1}{p_{0}^{2} \sin ^{2} \psi}\left[\frac{2 L}{p_{0} p_{1}}\left(2 \Gamma_{0}^{2}-\Gamma_{0} \Gamma_{1}-1-\frac{k}{\Delta}\right)\right. \\
& \left.\left.-\frac{4 \varepsilon^{T}\left(\Delta-\Gamma_{1}\right)^{2}}{p_{1} T}-\frac{2 \varepsilon\left(\Delta-\Gamma_{1}\right)}{p_{1}}\right]\right\} .
\end{aligned}
$$

In the above formulae, $Z$ is the ion charge, $r_{0}$ is the classical electron radius, $k$ is the normalized photon impulse

$k=\frac{h v}{m_{\mathrm{e}} c^{2}}$,

$\Gamma_{0}$ and $\Gamma_{1}$ are the normalized electron energies before and after scattering, respectively

$\Gamma_{0}=\frac{E}{m_{\mathrm{e}} c^{2}}+1, \quad \Gamma_{1}=\Gamma_{0}-k$,

$p_{0}$ and $p_{1}$ are the normalized electron impulses before and after scattering, respectively

$p_{0}=\sqrt{\Gamma_{0}^{2}-1}, \quad p_{1}=\sqrt{\Gamma_{1}^{2}-1}$,

and the angle $\psi$ is determined by Eq. (A.3). Other parameters in Eqs. (A.4), (A.5) are defined as

$T=\left|p_{0}-k\right|=\sqrt{p_{0}^{2}+k^{2}-2 p_{0} k \cos \psi}$,

$L=\ln \frac{\Gamma_{0} \Gamma_{1}-1+p_{0} p_{1}}{\Gamma_{0} \Gamma_{1}-1-p_{0} p_{1}}$

$\varepsilon=\ln \frac{\Gamma_{1}+p_{1}}{\Gamma_{1}-p_{1}}, \quad \varepsilon_{T}=\ln \frac{T+p_{1}}{T-p_{1}}$,

$\Delta=\Gamma_{0}-p_{0} \cos \psi$

The factor $Q_{E}$ is the Coulomb correction (Koch \& Motz 1959):

$Q_{E}=\frac{\beta_{0}}{\beta_{1}} \frac{1-\mathrm{e}^{-(2 \pi Z / 137) / \beta_{0}}}{1-\mathrm{e}^{-(2 \pi Z / 137) / \beta_{1}}}$,

where

$\beta_{0}=\frac{p_{0}}{\Gamma_{0}}, \quad \beta_{1}=\frac{p_{1}}{\Gamma_{1}}$. 


\section{References}

Aschwanden, M. J. 2005, Physics of the Solar Corona. An Introduction with Problems and Solutions, 2nd edn., ed. M. J. Aschwanden

Bai, T., \& Ramaty, R. 1978, ApJ, 219, 705

Bastian, T. S. 1999, in Proceedings of the Nobeyama Symposium, held in Kiyosato, Japan, Oct. 27-30, 1998, ed. T. S. Bastian, N. Gopalswamy, \& K. Shibasaki, NRO Rep., 479, 211

Bastian, T. S., Benz, A. O., \& Gary, D. E. 1998, ARA\&A, 36, 131

Battaglia, M., \& Benz, A. O. 2006, A\&A, 456, 751

Benz, A., 2002, Plasma Astrophysics, second edition, Ap\&SS Library, 279.

Benz, A. O. 1977, ApJ, 211, 270

Boggs, S. E., Coburn, W., \& Kalemci, E. 2006, ApJ, 638, 1129

Brown, J. C. 1971, Sol. Phys., 18, 489

Brown, J. C. 1972, Sol. Phys., 26, 441

Brown, J. C., \& Loran, J. M. 1985, MNRAS, 212, 245

Brown, J. C., Emslie, A. G., Holman, G. D., et al. 2006, ApJ, 643, 523

Diakonov, S. V., \& Somov, B. V. 1988, Sol. Phys., 116, 119

Elwert, G., \& Haug, E. 1970, Sol. Phys., 15, 234

Emslie, A. G. 1978, ApJ, 224, 241

Emslie, A. G. 1980, ApJ, 235, 1055

Fletcher, L., et al. 2010, Space Sci. Rev., submitted

Gluckstern, R. L., \& Hull, M. H. 1953, Phys. Rev., 90, 1030

Grigis, P. C., \& Benz, A. O. 2004, A\&A, 426, 1093

Holman, G. D., Aschwanden, M. J., Aurass, H., et al. 2010, Space Sci. Rev., subm.

Holman, G. D., Sui, L., Schwartz, R. A., \& Emslie, A. G. 2003, ApJ, 595, L97

Kašparová, J., Kontar, E. P., \& Brown, J. C. 2007, A\&A, 466, 705

Knight, J. W., \& Sturrock, P. A. 1977, ApJ, 218, 306

Koch, H. W., \& Motz, J. W. 1959, Rev. Mod. Phys., 31, 920

Kontar, E. P., \& Brown, J. C. 2006, ApJ, 653, L149

Kontar, E. P., MacKinnon, A. L., Schwartz, R. A., \& Brown, J. C. 2006, A\&A, 446, 1157

Kontar, E. P., Hannah, I. G., \& MacKinnon, A. L. 2008, A\&A, 489, L57

Krucker, S., Battaglia, M., Cargill, P. J., et al. 2008, A\&A Rev., 16, 155

Kundu, M. R., Grechnev, V. V., Garaimov, V. I., \& White, S. M. 2001a, ApJ, 563,389

Kundu, M. R., Nindos, A., White, S. M., \& Grechnev, V. V. 2001b, ApJ, 557, 880

Kundu, M. R., Nindos, A., \& Grechnev, V. V. 2004, A\&A, 420, 351
Kuznetsov, S. N., Kurt, V. G., Myagkova, I. N., Yushkov, B. Y., \& Kudela, K. 2006, Sol. Sys. Res., 40, 104

Landau, L. D. 1937, Zh. Eksp. Teor. Fiz., 7, 203

Leach, J., \& Petrosian, V. 1981, ApJ, 251, 781

Leach, J., \& Petrosian, V. 1983, ApJ, 269, 715

Lin, R. P., Krucker, S., Hurford, G. J., et al. 2003, ApJ, 595, L69

Masuda, S., Kosugi, T., Hara, H., Tsuneta, S., \& Ogawara, Y. 1994, Nature, 371, 495

McClements, K. G. 1992, A\&A, 253, 261

McConnell, M. L., Smith, D. M., Emslie, A. G., et al. 2003, in BAAS, 35, 850

Samarskii, A. A. 2001, Monographs and Textbooks in Pure and Applied Mathematics, The theory of difference schemes (New York: Marcel Dekker Inc.), 240, translated from the Russian

Simnett, G. M. 1995, Space Sci. Rev., 73, 387

Siversky, T. V., \& Zharkova, V. V. 2009, A\&A, in press

Spicer, D. S. 1977, Sol. Phys., 54, 379

Suarez-Garcia, E., Hajdas, W., Wigger, C., et al. 2006, Sol. Phys., 239, 149

Sui, L., Holman, G. D., \& Dennis, B. R. 2005, ApJ, 626, 1102

Sui, L., Holman, G. D., \& Dennis, B. R. 2007, ApJ, 670, 862

Sui, L., Holman, G. D., Dennis, B. R., et al. 2002, Sol. Phys., 210, 245

Tindo, I. P., Ivanov, V. D., Mandel'Stam, S. L., \& Shuryghin, A. I. 1970, Sol. Phys., 14, 204

Tindo, I. P., Ivanov, V. D., Mandel'Stam, S. L., \& Shuryghin, A. I. 1972a, Sol. Phys., 24, 429

Tindo, I. P., Ivanov, V. D., Valníček, B., \& Livshits, M. A. 1972b, Sol. Phys., 27, 426

Tindo, I. P., Shurygin, A. I., \& Steffen, W. 1976, Sol. Phys., 46, 219

Tramiel, L. J., Novick, R., \& Chanan, G. A. 1984, ApJ, 280, 440

van den Oord, G. H. J. 1990, A\&A, 234, 496

Vilmer, N., Krucker, S., Lin, R. P., \& The Rhessi Team 2002, Sol. Phys., 210, 261

White, S., et al. 2010, Space Sci. Rev., subm.

Willson, R. F., \& Holman, G. 2003, in BAAS, 35, 836

Zharkova, V. V., \& Kobylinskii, V. A. 1993, Sol. Phys., 143, 259

Zharkova, V. V., Azner, K., Benz, A., et al. 2010, Space Sci. Rev., submitted

Zharkova, V. V., Brown, J. C., \& Syniavskii, D. V. 1995, A\&A, 304, 284

Zharkova, V. V., \& Gordovskyy, M. 2005, A\&A, 432, 1033

Zharkova, V. V., \& Gordovskyy, M. 2006, ApJ, 651, 553

Zhitnik, I. A., Logachev, Y. I., Bogomolov, A. V., et al. 2006, Sol. Sys. Res., 40, 93 